\title{
The Structure of Essential Spectra and Discrete Spectrum of Three-Electron Systems in the Impurity Hubbard Model-Quartet State
}

\author{
S. M. Tashpulatov \\ Institute of Nuclear Physics of Academy of Science of Republic of Uzbekistan, Tashkent, Uzbekistan \\ Email: sadullatashpulatov@yandex.com, toshpul@mail.ru, toshpul@inp.uz
}

How to cite this paper: Tashpulatov, S.M. (2021) The Structure of Essential Spectra and Discrete Spectrum of Three-Electron Systems in the Impurity Hubbard Model-Quartet State. Journal of Applied Mathematics and Physics, 9, 1391-1421. https://doi.org/10.4236/jamp.2021.96094

Received: March 31, 2021

Accepted: June 27, 2021

Published: June 30, 2021

Copyright (c) 2021 by author(s) and Scientific Research Publishing Inc. This work is licensed under the Creative Commons Attribution International License (CC BY 4.0). http://creativecommons.org/licenses/by/4.0/

\begin{abstract}
We consider a three-electron system in the Impurity Hubbard model with a coupling between nearest-neighbors. Our research aim consists of studying the structure of essential spectrum and discrete spectra of the energy operator of three-electron systems in the impurity Hubbard model in the quartet state of the system in a $v$-dimensional lattice. We have reduced the study of the spectrum of the three-electron quartet state operator in the impurity Hubbard model to the study of the spectrum of a simpler operator. We proved the essential spectra of the three-electron systems in the Impurity Hubbard model in the quartet state is the union of no more than six segments, and the discrete spectrum of the system is consists of no more than four eigenvalues.
\end{abstract}

\section{Keywords}

Essential Spectrum, Discrete Spectrum, Three-Electron System, Local Impurity States, Impurity Hubbard Model, Quartet State, Doublet State

\section{Introduction}

In the early 1970s, three papers [1] [2] [3], where a simple model of metal was proposed that has become a fundamental model in the theory of strongly correlated electron systems, appeared almost simultaneously and independently. In that model, a single nondegenerate electron band with a local Coulomb interaction is considered. The model proposed in [1] [2] [3] was called the Hubbard model after John Hubbard, who made a fundamental contribution to studying the statistical mechanics of that system, although the local form of Coulomb interaction was first introduced for an impurity model in metal by Anderson [4]. We also recall that the Hubbard model is a particular case of the Shubin-Wonsowsky 
polaron model [5], which had appeared 30 years before [1] [2] [3]. In the Shubin-Wonsowsky model, along with the on-site Coulomb interaction, the interaction of electrons on neighboring sites is also taken into account. The Hubbard model is an approximation used in solid-state physics to describe the transition between conducting and insulating states. It is the simplest model describing particle interaction on a lattice.

The Hubbard model and impurity Hubbard model is currently one of the most extensively studied multielectron models of metals [6] [7] [8] [9] [10]. But little is known about exact results for the spectrum and wave functions of the crystal described by the Hubbard model, and obtaining the corresponding statements is therefore of great interest. The spectrum and wave functions of the system of two electrons in a crystal described by the Hubbard Hamiltonian were studied in [6]. It is known that two-electron systems can be in two states, triplet and singlet [6] [7] [8] [9] [10]. It was proved in [6] that the spectrum of the system Hamiltonian $H^{t}$ in the triplet state is purely continuous and coincides with a segment $[m, M]$, and the operator $H^{s}$ of the system in the singlet state, in addition to the continuous spectrum $[m, M]$, has a unique antibound state for some values of the quasimomentum. For the antibound state, correlated motion of the electrons is realized under which the contribution of binary states is large. Because the system is closed, the energy must remain constant and large. This prevents the electrons from being separated by long distances. Next, an essential point is that bound states (sometimes called scattering-type states) do not form below the continuous spectrum. This can be easily understood because the interaction is repulsive. We note that a converse situation is realized for $U<0$ : below the continuous spectrum, there is a bound state (antibound states are absent) because the electrons are then attracted to one another.

For the first band, the spectrum is independent of the parameter $U$ of the on-site Coulomb interaction of two electrons and corresponds to the energy of two noninteracting electrons, being exactly equal to the triplet band. The second band is determined by Coulomb interaction to a much greater degree: both the amplitudes and the energy of two electrons depend on $U$, and the band itself disappears as $U \rightarrow 0$ and increases without bound as $U \rightarrow \infty$. The second band largely corresponds to a one-particle state, namely, the motion of the doublet, i.e., two-electron bound states.

The spectrum and wave functions of the system of three electrons in a crystal described by the Hubbard Hamiltonian were studied in [11].

The spectrum of the energy operator of system of four electrons in a crystal described by the Hubbard Hamiltonian in the triplet state was studied in [12]. The four-electron system exists quintet state, and three type triplet states, and two type singlet states. The spectrum of the energy operator of four-electron systems in the Hubbard model in the quintet, and singlet states was studied in [13].

Here, we consider the energy operator of three-electron systems in the Impurity Hubbard model and describe the structure of the essential spectra and dis- 
crete spectrum of the system for quartet state.

The Hamiltonian of the chosen model has the form

$$
\begin{aligned}
H= & A \sum_{m, \gamma} a_{m, \gamma}^{+} a_{m, \gamma}+B \sum_{m, \tau, \gamma} a_{m, \gamma}^{+} a_{m+\tau, \gamma}+U \sum_{m} a_{m, \uparrow}^{+} a_{m, \uparrow} a_{m, \downarrow}^{+} a_{m, \downarrow} \\
& +\left(A_{0}-A\right) \sum_{\gamma} a_{0, \gamma}^{+} a_{0, \gamma}+\left(B_{0}-B\right) \sum_{\tau, \gamma}\left(a_{0, \gamma}^{+} a_{\tau, \gamma}+a_{\tau, \gamma}^{+} a_{0, \gamma}\right) \\
& +\left(U_{0}-U\right) a_{0, \uparrow}^{+} a_{0, \uparrow} a_{0, \downarrow}^{+} a_{0, \downarrow} \cdot
\end{aligned}
$$

Here $A\left(A_{0}\right)$ is the electron energy at a regular (impurity) lattice site, $B\left(B_{0}\right)$ is the transfer integral between (between electron and impurities) neighboring sites (we assume that $B>0 \quad\left(B_{0}>0\right)$ for convenience), $\tau= \pm e_{j}, j=1,2, \cdots, v$, where $e_{j}$ are unit mutually orthogonal vectors, which means that summation is taken over the nearest neighbors, $U\left(U_{0}\right)$ is the parameter of the on-site Coulomb interaction of two electrons in the regular (impurity) sites, $\gamma$ is the spin index, $\gamma=\uparrow$ or $\gamma=\downarrow, \uparrow$ and $\downarrow$ denote the spin values $\frac{1}{2}$ and $-\frac{1}{2}$, and $a_{m, \gamma}^{+}$ and $a_{m, \gamma}$ are the respective electron creation and annihilation operators at a site $m \in Z^{v}$.

The three electron systems have a quartet state and two type doublet states.

The energy of the system depends on its total spin $S$. Along with the Hamiltonian, the $N_{e}$ electron system is characterized by the total spin $S$,

$$
S=S_{\max }, S_{\max }-1, \cdots, S_{\min }, S_{\max }=\frac{N_{e}}{2}, S_{\min }=0, \frac{1}{2} \text {. }
$$

Hamiltonian (1) commutes with all components of the total spin operator $S=\left(S^{+}, S^{-}, S^{z}\right)$, and the structure of eigenfunctions and eigenvalues of the system therefore depends on $S$. The Hamiltonian $H$ acts in the antisymmetric Fock space $\mathscr{H}_{a s}$.

\section{Quartet State}

Let $\varphi_{0}$ be the vacuum vector in the space $\mathscr{K}_{a s}$. The quartet state corresponds to the free motion of three electrons over the lattice with the basic functions $q_{m, n, p \in Z^{v}}^{3 / 2}=a_{m, \uparrow}^{+} a_{n, \uparrow}^{+} a_{p, \uparrow}^{+} \varphi_{0}$. The space $H_{3 / 2}^{q}$, corresponding to the quartet state is the set of all vectors of the form $\psi_{3 / 2}^{q}=\sum_{m, n, p \in Z^{v}} f(m, n, p) q_{m, n, p \in Z^{v}}^{3 / 2}, f \in l_{2}^{a s}$, where $l_{2}^{a s}$ is the space of antisymmetric functions in the space $l_{2}\left(\left(Z^{v}\right)^{3}\right)$.

Theorem 1. The space $\mathscr{Y}_{3 / 2}^{q}$ is invariant under the operator $H$, and the restriction $H_{3 / 2}^{q}$ of operator $H$ to the subspace $\mathscr{H}_{3 / 2}^{q}$ is a bounded self-adjoint operator. It generates a bounded self-adjoint operator $\bar{H}_{3 / 2}^{q}$ acting in the space $l_{2}^{a s}\left(\left(Z^{v}\right)^{3}\right)$ according to the formula

$$
\begin{aligned}
\left(\bar{H}_{3 / 2}^{q} f\right)(p, r, t)= & 3 A f(p, r, t)+2 B \sum_{\tau}[f(p+\tau, r, t)+f(p, r+\tau, t) \\
& +f(p, r, t+\tau)]+\left(A_{0}-A\right)\left[\delta_{p, 0}+\delta_{r, 0}+\delta_{t, 0}\right] f(p, r, t) \\
& +\left(B_{0}-B\right) \sum_{\tau}\left[\delta_{p, \tau} f(0, r, t)+\delta_{r, \tau} f(p, 0, t)+\delta_{t, \tau} f(p, r, 0)\right. \\
& \left.+\delta_{p, 0} f(\tau, r, t)+\delta_{r, 0} f(p, \tau, t)+\delta_{t, 0} f(p, r, \tau)\right],
\end{aligned}
$$


where $\delta_{k, j}$ is the Kronecker symbol. The operator $H_{3 / 2}^{q}$ acts on a vector $\psi_{3 / 2}^{q} \in \mathscr{H}_{3 / 2}^{q}$ according to the formula

$$
H_{3 / 2}^{q} \psi_{3 / 2}^{q}=\sum_{p, r, t \in Z^{v}}\left(\bar{H}_{3 / 2}^{q} f\right)(p, r, t) q_{p, r, t \in Z^{v}}^{3 / 2} .
$$

\section{Proof.}

We act with the Hamiltonian $H$ on vectors $\psi_{3 / 2}^{q} \in \mathscr{H}_{3 / 2}^{q}$ using the standard anticommutation relations between electron creation and annihilation operators at lattice sites, $\left\{a_{m, \gamma}, a_{n, \beta}^{+}\right\}=\delta_{m, n} \delta_{\gamma, \beta},\left\{a_{m, \gamma}, a_{n, \beta}\right\}=\left\{a_{m, \gamma}^{+}, a_{n, \beta}^{+}\right\}=\theta$, and also take into account that $a_{m, \gamma} \varphi_{0}=\theta$, where $\theta$ is the zero element of $\mathscr{Y}_{3 / 2}^{q}$. This yields the statement of the theorem.

We let $\varepsilon_{1}=A_{0}-A$, and $\varepsilon_{2}=B_{0}-B$.

Lemma 1. The spectra of the operators $H_{3 / 2}^{q}$ and $\bar{H}_{3 / 2}^{q}$ coincide.

Proof. Because the operators $H_{3 / 2}^{q}$ and $\bar{H}_{3 / 2}^{q}$ are bounded self-adjoint operators, it follows that if $\lambda \in \sigma\left(H_{3 / 2}^{q}\right)$, then the Weyl criterion (see [14], chapter VII, paragraph 3, pp. 262-263) implies that there is a sequence $\left\{\psi_{n}\right\}_{n=1}^{\infty}$ such that $\left\|\psi_{n}\right\|=1$ and $\lim _{n \rightarrow \infty}\left\|\left(H_{3 / 2}^{q}-\lambda\right) \psi_{n}\right\|=0$. We set

$$
\begin{aligned}
& \psi_{n}=\sum_{p, r, t} f_{n}(p, r, t) a_{p, \uparrow}^{+} a_{r, \uparrow}^{+} a_{t, \uparrow}^{+} \varphi_{0} \text {. Then } \\
& \left\|\left(H_{3 / 2}^{q}-\lambda\right) \psi_{n}\right\|^{2}=\left(\left(H_{3 / 2}^{q}-\lambda\right) \psi_{n},\left(H_{3 / 2}^{q}-\lambda\right) \psi_{n}\right) \\
& =\sum_{p, r, t}\left\|\left(\bar{H}_{3 / 2}^{q}-\lambda\right) f_{n}(p, r, t)\right\|^{2}\left(a_{p, \uparrow}^{+} a_{r, \uparrow}^{+} a_{t, \uparrow}^{+} \varphi_{0}, a_{p, \uparrow}^{+} a_{r, \uparrow}^{+} a_{t, \uparrow}^{+} \varphi_{0}\right) \\
& =\sum_{p, r, t}\left\|\left(\bar{H}_{3 / 2}^{q}-\lambda\right) F_{n}(p, r, t)\right\|^{2}\left(a_{t, \uparrow} a_{r, \uparrow} a_{p, \uparrow} a_{p, \uparrow}^{+} a_{r, \uparrow}^{+} a_{t, \uparrow}^{+} \varphi_{0}, \varphi_{0}\right) \text {, as } \\
& =\sum_{p, r, t}\left\|\left(\bar{H}_{3 / 2}^{q}-\lambda\right) F_{n}(p, r, t)\right\|^{2}\left(\varphi_{0}, \varphi_{0}\right) \\
& =\sum_{p, r, t}\left\|\left(\bar{H}_{3 / 2}^{q}-\lambda\right) F_{n}(p, r, t)\right\|^{2} \rightarrow 0
\end{aligned}
$$

$n \rightarrow \infty$, where $F_{n}=\sum_{p, r, t} f_{n}(p, r, t)$. It follows that $\lambda \in \sigma\left(\bar{H}_{3 / 2}^{q}\right)$. Consequently, $\sigma\left(H_{3 / 2}^{q}\right) \subset \sigma\left(\bar{H}_{3 / 2}^{q}\right)$.

Conversely, let $\bar{\lambda} \in \sigma\left(\bar{H}_{3 / 2}^{q}\right)$. Then, by the Weyl criterion, there is a sequence $\left\{F_{n}\right\}_{n=1}^{\infty}$ such that $\left\|F_{n}\right\|=1$ and $\lim _{n \rightarrow \infty}\left\|\left(\bar{H}_{3 / 2}^{q}-\bar{\lambda}\right) \psi_{n}\right\|=0$. Setting

$F_{n}=\sum_{p, r, t} f_{n}(p, r, t),\left\|F_{n}\right\|=\left(\sum_{p, r, t}\left|f_{n}(p, r, t)\right|^{2}\right)^{\frac{1}{2}}$, we conclude that $\left\|\psi_{n}\right\|=\left\|F_{n}\right\|=1$ and $\left\|\left(\bar{H}_{3 / 2}^{q}-\bar{\lambda}\right) F_{n}\right\|=\left\|\left(\bar{H}_{3 / 2}^{q}-\bar{\lambda}\right) \psi_{n}\right\| \rightarrow 0$ as $n \rightarrow \infty$. This means that $\bar{\lambda} \in \sigma\left(H_{3 / 2}^{q}\right)$ and hence $\sigma\left(\bar{H}_{3 / 2}^{q}\right) \subset \sigma\left(H_{3 / 2}^{q}\right)$. These two relations imply $\sigma\left(H_{3 / 2}^{q}\right)=\sigma\left(\bar{H}_{3 / 2}^{q}\right)$.

We call the operator $H_{3 / 2}^{q}$ the three-electron quartet state operator.

Let $\mathscr{T}: l_{2}\left(\left(Z^{v}\right)^{3}\right) \rightarrow L_{2}\left(\left(T^{v}\right)^{3}\right) \equiv \tilde{\mathscr{X}}_{3 / 2}^{q}$ be the Fourier transform, where $T^{v}$ is the $v$-dimensional torus endowed with the normalized Lebesgue measure d $\lambda$, i.e. $\lambda\left(T^{v}\right)=1$.

We set $\tilde{H}_{3 / 2}^{q}=\mathscr{T} \bar{H}_{3 / 2}^{q} \mathscr{T}^{-1}$. In the quasimomentum representation, the operator $\bar{H}_{3 / 2}^{q}$ acts in the Hilbert space $L_{2}^{a s}\left(\left(Z^{v}\right)^{3}\right)$, where $L_{2}^{a s}\left(\left(T^{v}\right)^{3}\right)$ is the sub- 
space of antisymmetric functions in $L_{2}\left(\left(T^{v}\right)^{3}\right)$.

Theorem 2. The Fourier transform of operator $\bar{H}_{3 / 2}^{q}$ is a bounded self-adjoint operator $\tilde{H}_{3 / 2}^{q}=\mathscr{F} \bar{H}_{3 / 2}^{q} \mathscr{F}^{-1}$ acting in the space $\tilde{\mathscr{C}}_{3 / 2}^{q}$ according to the formula

$$
\begin{aligned}
\tilde{H}_{3 / 2}^{q} \psi_{3 / 2}^{q}= & 3 A f(\lambda, \mu, \gamma)+2 B \sum_{i=1}^{v}\left[\cos \left(\lambda_{i}\right)+\cos \left(\mu_{i}\right)+\cos \left(\gamma_{i}\right)\right] f(\lambda, \mu, \gamma) \\
& +\varepsilon_{1}\left[\int_{T^{v}} f(s, \mu, \gamma) \mathrm{d} s+\int_{T^{v}} f(\lambda, t, \gamma) \mathrm{d} t+\int_{T^{v}} f(\lambda, \mu, l) \mathrm{d} l\right] \\
& +2 \varepsilon_{2} \int_{T^{v}} \sum_{i=1}^{v}\left[\cos \left(\lambda_{i}\right)+\cos \left(s_{i}\right)\right] f(s, \mu, \gamma) \mathrm{d} s \\
& +2 \varepsilon_{2} \int_{T^{v}} \sum_{i=1}^{v}\left[\cos \left(\mu_{i}\right)+\cos \left(t_{i}\right)\right] f(\lambda, t, \gamma) \mathrm{d} t \\
& +2 \varepsilon_{2} \int_{T^{v}} \sum_{i=1}^{v}\left[\cos \left(\gamma_{i}\right)+\cos \left(l_{i}\right)\right] f(\lambda, \mu, l) \mathrm{d} l .
\end{aligned}
$$

The proof of Theorem 2, is straightforward of (2) using the Fourier transformation.

It is clear that spectral properties of energy operator of three-electron systems in the impurity Hubbard model in the quartet state are closely related to the spectral properties of its one-electron subsystems in the impurity Hubbard model. First we investigate the spectrum of one-electron subsystems.

\section{Spectra of the Energy Operator of One-Electron System in the Impurity Hubbard Model}

The Hamiltonian $H$ of one-electron systems in the impurity Hubbard model also has the form (1). We let $\mathscr{H}_{1}$ denote the Hilbert space spanned by the vectors in the form $\psi=\sum_{p} a_{p, \uparrow}^{+} \varphi_{0}$. It is called the space of one-electron states of the operator $H$. The space $\mathscr{H}_{1}$ is invariant with respect to action of the operator $H$. Denote by $H_{1}=\left.H\right|_{\mathscr{R}_{1}}$ the restriction of $H$ to the subspace $\mathscr{H}_{1}$.

As in the proof of Theorem 3, using the standard anticommutation relations between electron creation and annihilation operators at lattice sites, we get the following.

Theorem 3. The subspace $\mathscr{H}_{1}$ is invariant with respect to the action of the operator $H$, and the restriction $H_{1}$ is a linear bounded self-adjoint operator, acting in $\mathscr{H}_{1}$ as

$$
H_{1} \psi=\sum_{p}\left(\bar{H}_{1} f\right)(p) a_{p, \uparrow}^{+} \varphi_{0}, \psi \in \mathscr{H}_{1},
$$

where $\bar{H}_{1}$ is a linear bounded self-adjoint operator acting in the space $l_{2}$ as $\left(\bar{H}_{1} f\right)(p)=A f(p)+B \sum_{\tau} f(p+\tau)+\varepsilon_{1} \delta_{p, 0} f(p)+\varepsilon_{2} \sum_{\tau}\left(\delta_{p, \tau} f(0)+\delta_{p, 0} f(\tau)\right) .(6)$

Lemma 2. The spectra of the operators $\bar{H}_{1}$ and $H_{1}$ coincide. The proof of Lemma 2 is the same as the proof of the Lemma 1.

As in Section 2 denote by $\mathscr{F}: l_{2}\left(Z^{v}\right) \rightarrow L_{2}\left(T^{v}\right):=\tilde{\mathscr{H}}_{1}$ the Fourier transform. Setting $\tilde{H}_{1}=\mathscr{F} \bar{H}_{1} \mathscr{F}^{-1}$ we get that the operator $\bar{H}_{1}$ acts in the Hilbert space $L_{2}\left(T^{v}\right)$. 
Using the equality (6) and properties of the Fourier transform we have the following.

Theorem 4. The operator $\tilde{H}_{1}$ acting in the space $\tilde{H}_{1}$ as

$$
\begin{aligned}
& \left(\tilde{H}_{1} f\right)(\mu)=\left[A+2 B \sum_{i=1}^{v} \cos \mu_{i}\right] f(\mu)+\varepsilon_{1} \int_{T^{v}} f(s) \mathrm{d} s \\
& +2 \varepsilon_{2} \int_{T^{v}} \sum_{i=1}^{v}\left[\cos \mu_{i}+\cos s_{i}\right] f(s) \mathrm{d} s, \mu=\left(\mu_{1}, \cdots, \mu_{n}\right), s=\left(s_{1}, \cdots, s_{n}\right) \in T^{v} .
\end{aligned}
$$

It is clear that the continuous spectrum of operator $\tilde{H}_{1}$ is independent of the numbers $\varepsilon_{1}$ and $\varepsilon_{2}$, and is equal to segment $\left[m_{v}, M_{v}\right]=[A-2 B v, A+2 B v]$, where $m_{v}=\min _{x \in T^{v}} h(x), \quad M_{v}=\max _{x \in T^{v}} h(x)$ (here $\left.h(x)=A+2 B \sum_{i=1}^{v} \cos x_{i}\right)$.

To find the eigenvalues and eigenfunctions of operator $\tilde{H}_{1}$ we rewrite (7) in following form:

$$
\begin{aligned}
& \left\{A+2 B \sum_{i=1}^{v} \cos \mu_{i}-z\right\} f(\mu)+\varepsilon_{1} \int_{T^{v}} f(s) \mathrm{d} s \\
& +2 \varepsilon_{2} \int_{T^{v}} \sum_{i=1}^{v}\left[\cos \mu_{i}+\cos s_{i}\right] f(s) \mathrm{d} s=0,
\end{aligned}
$$

where $z \in \mathbb{R}$.

Suppose first that $v=1$ and denote $a=\int_{T} f(s) \mathrm{d} s, \quad b=\int_{T} f(s) \cos s \mathrm{~d} s$, $h(\mu)=A+2 B \cos \mu$. From (8) it follows that

$$
f(\mu)=-\frac{\left(\varepsilon_{1}+2 \varepsilon_{2} \cos \mu\right) a+2 \varepsilon_{2} b}{h(\mu)-z} .
$$

Now substitute (9) in expressing of $a$ and $b$ we get the following system of two linear homogeneous algebraic equations:

$$
\begin{gathered}
\left(1+\int_{T} \frac{\varepsilon_{1}+2 \varepsilon_{2} \cos s}{h(s)-z} \mathrm{~d} s\right) \cdot a+2 \varepsilon_{2} \int_{T} \frac{\mathrm{d} s}{h(s)-z} \cdot b=0 ; \\
\int_{T} \frac{\cos s\left(\varepsilon_{1}+2 \varepsilon_{2} \cos s\right)}{h(s)-z} \mathrm{~d} s \cdot a+\left(1+2 \varepsilon_{2} \int_{T} \frac{\cos s \mathrm{~d} s}{h(s)-z}\right) \cdot b=0 .
\end{gathered}
$$

This system has a nontrivial solution if and only if the determinant $\Delta_{1}(z)$ of this system is equal to zero, where

$$
\begin{aligned}
\Delta_{1}(z)= & \left(1+\int_{T} \frac{\left(\varepsilon_{1}+2 \varepsilon_{2} \cos s\right) \mathrm{d} s}{h(s)-z}\right) \cdot\left(1+2 \varepsilon_{2} \int_{T} \frac{\cos s \mathrm{~d} s}{h(s)-z}\right) \\
& -2 \varepsilon_{2} \int_{T} \frac{\mathrm{d} s}{h(s)-z} \cdot \int_{T} \frac{\cos s\left(\varepsilon_{1}+2 \varepsilon_{2} \cos s\right)}{h(s)-z} \mathrm{~d} s .
\end{aligned}
$$

Therefore, it is true the following.

Lemma 3. If a real number $z \notin\left[m_{1}, M_{1}\right]$ then $z$ is an eigenvalue of the operator $\tilde{H}_{1}$ if and only if $\Delta_{1}(z)=0$.

The following Theorem describe of the exchange of the spectrum of operator $\tilde{H}_{1}$ in the case $v=1$.

Theorem 5. Let $v=1$. Then

1) If $\varepsilon_{2}=-B$ and $\varepsilon_{1}<-2 B$ (respectively, $\varepsilon_{2}=-B$ and $\varepsilon_{1}>2 B$,) then 
the operator $\tilde{H}_{1}$ has a unique eigenvalue $z=A+\varepsilon_{1}$, lying the below (respectively, above) of the continuous spectrum of the operator $\tilde{H}_{1}$.

2) If $\varepsilon_{2}=-2 B$ and $\varepsilon_{1}<0$ (respectively, $\varepsilon_{2}=-2 B$ and $\varepsilon_{1}>0$ ), then the operator $\tilde{H}_{1}$ has a unique eigenvalue $z=A-\sqrt{4 B^{2}+\varepsilon_{1}^{2}}$ (respectively, $z=A+\sqrt{4 B^{2}+\varepsilon_{1}^{2}}$ ), lying the below (respectively, above) of the continuous spectrum of the operator $\tilde{H}_{1}$.

3) If $\varepsilon_{2}=0$ and $\varepsilon_{1}<0$ (respectively, $\varepsilon_{2}=0$ and $\varepsilon_{1}>0$ ), then the operator $\tilde{H}_{1}$ has a unique eigenvalue $\mathrm{z}=A-\sqrt{4 B^{2}+\varepsilon_{1}^{2}}$ (respectively, $z=A+\sqrt{4 B^{2}+\varepsilon_{1}^{2}}$ ), lying the below (respectively, above) of the continuous spectrum of the operator $\tilde{H}_{1}$.

4) If $\varepsilon_{1}=0$ and $\varepsilon_{2}>0$ (respectively, $\varepsilon_{1}=0$ and $\varepsilon_{2}<-2 B$ ), then the operator $\tilde{H}_{1}$ has a unique eigenvalue $z=A-\frac{2 B E}{\sqrt{E^{2}-1}},\left(z=A+\frac{2 B E}{\sqrt{E^{2}-1}}\right)$, where $E=\frac{\left(B+\varepsilon_{2}\right)^{2}}{\varepsilon_{2}^{2}+2 B \varepsilon_{2}}$, lying the below (above) of the continuous spectrum of the operator $\tilde{H}_{1}$.

5) If $\varepsilon_{1}=\frac{2\left(\varepsilon_{2}^{2}+2 B \varepsilon_{2}\right)}{B}$, (respectively, $\left.\varepsilon_{1}=-\frac{2\left(\varepsilon_{2}^{2}+2 B \varepsilon_{2}\right)}{B}\right)$, then the operator $\tilde{H}_{1}$ has a unique eigenvalue $z=A+\frac{2 B\left(E^{2}+1\right)}{E^{2}-1}$, (respectively, $\left.Z=A-\frac{2 B\left(E^{2}+1\right)}{E^{2}-1}\right)$, where $E=\frac{\left(B+\varepsilon_{2}\right)^{2}}{\varepsilon_{2}^{2}+2 B \varepsilon_{2}}$, lying the above (respectively, below) of the continuous spectrum of the operator $\tilde{H}_{1}$.

6) If $\varepsilon_{2}>0$ and $\varepsilon_{1}>\frac{2\left(\varepsilon_{2}^{2}+2 B \varepsilon_{2}\right)}{B}$ (respectively, $\varepsilon_{2}<-2 B$ and $\left.\varepsilon_{1}>\frac{2\left(\varepsilon_{2}^{2}+2 B \varepsilon_{2}\right)}{B}\right)$, then the operator $\tilde{H}_{1}$ has a unique eigenvalue $z_{1}=A+\frac{2 B\left(\alpha+E \sqrt{E^{2}-1+\alpha^{2}}\right)}{E^{2}-1}$, where $E=\frac{\left(B+\varepsilon_{2}\right)^{2}}{\varepsilon_{2}^{2}+2 B \varepsilon_{2}}$, and the real number $\alpha>1$, lying the above of the continuous spectrum of the operator $\tilde{H}_{1}$.

7) If $\varepsilon_{2}>0$ and $\varepsilon_{1}<-\frac{2\left(\varepsilon_{2}^{2}+2 B \varepsilon_{2}\right)}{B}$ (respectively, $\varepsilon_{2}<-2 B$ and $\left.\varepsilon_{1}<-\frac{2\left(\varepsilon_{2}^{2}+2 B \varepsilon_{2}\right)}{B}\right)$, then the operator $\tilde{H}_{1}$ has a unique eigenvalue $z_{1}=A-\frac{2 B\left(\alpha+E \sqrt{E^{2}-1+\alpha^{2}}\right)}{E^{2}-1}<m_{1}$, where $E=\frac{\left(B+\varepsilon_{2}\right)^{2}}{\varepsilon_{2}^{2}+2 B \varepsilon_{2}}$, and the real number $\alpha>1$, lying the below of the continuous spectrum of the operator $\tilde{H}_{1}$.

8) If $\varepsilon_{2}>0$ and $-\frac{2\left(\varepsilon_{2}^{2}+2 B \varepsilon_{2}\right)}{B}<\varepsilon_{1}<\frac{2\left(\varepsilon_{2}^{2}+2 B \varepsilon_{2}\right)}{B}$ (respectively, $\varepsilon_{2}<-2 B$ and $\left.-\frac{2\left(\varepsilon_{2}^{2}+2 B \varepsilon_{2}\right)}{B}<\varepsilon_{1}<\frac{2\left(\varepsilon_{2}^{2}+2 B \varepsilon_{2}\right)}{B}\right)$, then the operator $\tilde{H}_{1}$ has 
a exactly two eigenvalues $z_{1}=A+\frac{2 B\left(\alpha+E \sqrt{E^{2}-1+\alpha^{2}}\right)}{E^{2}-1}<m_{1}$, and $z_{2}=A+\frac{2 B\left(\alpha-E \sqrt{E^{2}-1+\alpha^{2}}\right)}{E^{2}-1}>M_{1}$, where $E=\frac{\left(B+\varepsilon_{2}\right)^{2}}{\varepsilon_{2}^{2}+2 B \varepsilon_{2}}$, and the real number $|\alpha|<1$, lying the above and below of the continuous spectrum of the operator $\tilde{H}_{1}$.

9) If $-2 B<\varepsilon_{2}<0$, then the operator $\tilde{H}_{1}$ has no eigenvalues lying the outside of the continuous spectrum of the operator $\tilde{H}_{1}$.

Proof. In the case $v=1$, the continuous spectrum of the operator $\tilde{H}_{1}$ coincide with segment $\left[m_{1}, M_{1}\right]=[A-2 B, A+2 B]$. Expressing all integrals in the equation $\Delta_{1}(z)=0$ through the integral $J(z)=\int_{T} \frac{\mathrm{d} s}{A+2 B \cos s-z}$, we find that the equation $\Delta_{1}(z)=0$ is equivalent to the equation

$$
\left[\varepsilon_{1} B^{2}+\left(\varepsilon_{2}^{2}+2 B \varepsilon_{2}\right)(z-A)\right] J(z)+\left(B+\varepsilon_{2}\right)^{2}=0 .
$$

Moreover, the function $J(z)=\int_{T} \frac{\mathrm{d} s}{A+2 B \cos s-z}$ is a differentiable function on the set $\mathbb{R} \backslash\left[m_{1}, M_{1}\right]$, in addition,

$J^{\prime}(z)=\int_{T} \frac{\mathrm{d} s}{[A+2 B \cos s-z]^{2}}>0, z \notin\left[m_{1}, M_{1}\right]$. Thus the function $J(z)$ is an monotone increasing function on $\left(-\infty, m_{1}\right)$ and on $\left(M_{1},+\infty\right)$. Furthermore, $J(z) \rightarrow+0$ as $z \rightarrow-\infty, \quad J(z) \rightarrow+\infty$ as $z \rightarrow m_{1}-0, J(z) \rightarrow-\infty$ as $z \rightarrow M_{1}+0$, and $J(z) \rightarrow-0$ as $z \rightarrow+\infty$.

If $\varepsilon_{1} B^{2}+\left(\varepsilon_{2}^{2}+2 B \varepsilon_{2}\right)(z-A) \neq 0$ then from (13) follows that

$$
J(z)=-\frac{\left(B+\varepsilon_{2}\right)^{2}}{\varepsilon_{1} B^{2}+\left(\varepsilon_{2}^{2}+2 B \varepsilon_{2}\right)(z-A)} .
$$

The function $\psi(z)=-\frac{\left(B+\varepsilon_{2}\right)^{2}}{\varepsilon_{1} B^{2}+\left(\varepsilon_{2}^{2}+2 B \varepsilon_{2}\right)(z-A)}$ has a point of asymptotic discontinuity $z_{0}=A-\frac{B^{2} \varepsilon_{1}}{\varepsilon_{2}^{2}+2 B \varepsilon_{2}}$. Since $\psi^{\prime}(z)=\frac{\left(B+\varepsilon_{2}\right)^{2}\left(\varepsilon_{2}^{2}+2 B \varepsilon_{2}\right)}{\left[\varepsilon_{1} B^{2}+\left(\varepsilon_{2}^{2}+2 B \varepsilon_{2}\right)(z-A)\right]^{2}}$ for all $z \neq z_{0}$ it follows that the function $\psi(z)$ is an monotone increasing (decreasing) function on $\left(-\infty, z_{0}\right)$ and on $\left(z_{0},+\infty\right)$ in the case $\varepsilon_{2}^{2}+2 B \varepsilon_{2}>0$ (respectively, $\varepsilon_{2}^{2}+2 B \varepsilon_{2}<0$ ), in addition, and if $\varepsilon_{2}>0$, or $\varepsilon_{2}<-2 B$, then $\psi(z) \rightarrow+0$ as $z \rightarrow-\infty, \psi(z) \rightarrow+\infty$ as $z \rightarrow z_{0}-0, \psi(z) \rightarrow-\infty$ as $z \rightarrow z_{0}+0, \psi(z) \rightarrow-0$ as $z \rightarrow+\infty$ (respectively, if $-2 B<\varepsilon_{2}<0$, then $\psi(z) \rightarrow-0$ as $z \rightarrow-\infty, \psi(z) \rightarrow-\infty$ as $z \rightarrow z_{0}-0, \psi(z) \rightarrow+\infty$ as $z \rightarrow z_{0}+0, \psi(z) \rightarrow+0$ as $\left.z \rightarrow+\infty\right)$.

1) If $\varepsilon_{2}=-B$ and $\varepsilon_{1}<-2 B$ (respectively, $\varepsilon_{2}=-B$ and $\varepsilon_{1}>2 B$ ), then the equation for eigenvalues and eigenfunctions (13) has the form

$$
\left\{\varepsilon_{1} B^{2}-B^{2}(z-A)\right\} J(z)=0 .
$$

It is clear, that $J(z) \neq 0$ for the values $z \notin \sigma_{\text {cont }}\left(\tilde{H}_{1}\right)$. Therefore, 
$\varepsilon_{1}-z+A=0$, i.e., $z=A+\varepsilon_{1}$. If $\varepsilon_{1}<-2 B$, then this eigenvalue lying the below of the continuous spectrum of operator $\tilde{H}_{1}$, if $\varepsilon_{1}>2 B$, then this eigenvalue lying the above of the continuous spectrum of operator $\tilde{H}_{1}$.

2) If $\varepsilon_{2}=-2 B$ and $\varepsilon_{1}<0$ (respectively, $\varepsilon_{2}=-2 B$ and $\varepsilon_{1}>0$ ), then the equation for the eigenvalues and eigenfunctions has the form $\varepsilon_{1} B^{2} J(z)+B^{2}=0$, that is, $J(z)=-\frac{1}{\varepsilon_{1}}$. It is clear, what the integral $J(z)$ calculated in a quadrature, of the below (above) of continuous spectrum of operator $\tilde{H}_{1}$, the integral $J(z)>0,\left(J(z)<0\right.$, ) consequently, $\varepsilon_{1}<0 \quad\left(\varepsilon_{1}>0\right.$.) The calculated the integral $J(z)=\int_{T^{V}} \frac{\mathrm{d} s}{A+2 B \cos s-z}$, the below of the continuous spectrum of operator $\tilde{H}_{1}$, we have the equation of the form

$$
\frac{1}{\sqrt{(A-z)^{2}-4 B^{2}}}=-\frac{1}{\varepsilon_{1}} \text {. }
$$

This equation has a solution $z=A-\sqrt{\varepsilon_{1}^{2}+4 B^{2}}$, lying the below of the continuous spectrum of operator $\tilde{H}_{1}$. In the above of continuous spectrum of operator $\tilde{H}_{1}$, the equation take the form

$$
-\frac{1}{\sqrt{(z-A)^{2}-4 B^{2}}}=-\frac{1}{\varepsilon_{1}} .
$$

This equation has a solution of the form $z=A+\sqrt{\varepsilon_{1}^{2}+4 B^{2}}$, lying the above of the continuous spectrum of operator $\tilde{H}_{1}$.

3) If $\varepsilon_{2}=0$ and $\varepsilon_{1}<0$ (respectively, $\varepsilon_{2}=0$ and $\varepsilon_{1}>0$ ), then the equation for the eigenvalues and eigenfunctions take in the form $\varepsilon_{1} B^{2} J(z)+B^{2}=0$ or

$$
J(z)=-\frac{1}{\varepsilon_{1}} .
$$

It is clear, what the integral $J(z)$ calculated in a quadrature, of the below (respectively, above) of continuous spectrum of operator $\tilde{H}_{1}$, the integral $J(z)>0$, (respectively, $J(z)<0$,) consequently, $\varepsilon_{1}<0$ (respectively, $\varepsilon_{1}>0$.) The calculated the integral $J(z)=\int_{T^{v}} \frac{\mathrm{d} s}{A+2 B \cos s-z}$, the below of the continuous spectrum of operator $\tilde{H}_{1}$, we have the equation of the form

$$
\frac{1}{\sqrt{(A-z)^{2}-4 B^{2}}}=-\frac{1}{\varepsilon_{1}} \text {. }
$$

This equation has a solution $z=A-\sqrt{\varepsilon_{1}^{2}+4 B^{2}}$, lying the below of the continuous spectrum of operator $\tilde{H}_{1}$. In the above of continuous spectrum of operator $\tilde{H}_{1}$, the equation take the form

$$
-\frac{1}{\sqrt{(z-A)^{2}-4 B^{2}}}=-\frac{1}{\varepsilon_{1}} .
$$

This equation has a solution of the form $z=A+\sqrt{\varepsilon_{1}^{2}+4 B^{2}}$, lying the above 
of the continuous spectrum of operator $\tilde{H}_{1}$.

4) If $\varepsilon_{1}=0$ and $\varepsilon_{2}>0$ (respectively, $\varepsilon_{1}=0$ and $\varepsilon_{2}<-2 B$ ), then the equation for the eigenvalues and eigenfunctions take in the form

$$
\left(\varepsilon_{2}^{2}+2 B \varepsilon_{2}\right)(z-A) J(z)=-\left(B+\varepsilon_{2}\right)^{2},
$$

or

$$
J(z)=-\frac{\left(B+\varepsilon_{2}\right)^{2}}{\left(\varepsilon_{2}^{2}+2 B \varepsilon_{2}\right)(z-A)} .
$$

Denote $E=\frac{\left(B+\varepsilon_{2}\right)^{2}}{\varepsilon_{2}^{2}+2 B \varepsilon_{2}}$. Then $J(z)=-\frac{E}{z-A}$, or $J(z)=\frac{E}{A-z}$. In the below of the continuous spectrum of the operator $\tilde{H}_{1}$, we have the equation of the form

$$
\frac{1}{\sqrt{(A-z)^{2}-4 B^{2}}}=\frac{E}{A-z} \text {. }
$$

This equation has a solution $z=A-\frac{2 B E}{\sqrt{E^{2}-1}}$. It is obviously, that $E^{2}>1$.

This eigenvalue lying the below of the continuous spectrum of operator $\tilde{H}_{1}$. In the above of the continuous spectrum of operator $\tilde{H}_{1}$, the equation for the eigenvalues and eigenfunctions has the form

$$
-\frac{1}{\sqrt{(z-A)^{2}-4 B^{2}}}=-\frac{E}{z-A} .
$$

From here, we find $z=A+\frac{2 B E}{\sqrt{E^{2}-1}}$. This eigenvalue lying the above of the continuous spectrum of operator $\tilde{H}_{1}$.

5) If $\varepsilon_{1}=\frac{2\left(\varepsilon_{2}^{2}+2 B \varepsilon_{2}\right)}{B}$, then the equation for eigenvalues and eigenfunctions has the form

$$
\left(\varepsilon_{2}^{2}+2 B \varepsilon_{2}\right)(z-A+2 B) J(z)=-\left(B+\varepsilon_{2}\right)^{2},
$$

from this

$$
J(z)=-\frac{\left(B+\varepsilon_{2}\right)^{2}}{\left(\varepsilon_{2}^{2}+2 B \varepsilon_{2}\right)(z-A+2 B)} .
$$

We denote $E=\frac{\left(B+\varepsilon_{2}\right)^{2}}{\varepsilon_{2}^{2}+2 B \varepsilon_{2}}$. In the first we consider the Equation (25) in the below of continuous spectrum of operator $\tilde{H}_{1}$. In Equation (25) we find the equation of the form

$$
\frac{1}{\sqrt{(A-z)^{2}-4 B^{2}}}=\frac{E}{A-z-2 B} \text {. }
$$

From this, we find $z_{1}=A+\frac{2 B\left(E^{2}+1\right)}{E^{2}-1}$, and $z_{2}=A-2 B$. Now we verify the 
conditions $z_{i}<A-2 B, i=1,2$. The inequality $z_{1}<A-2 B$, is incorrectly, and inequality $z_{2}<A-2 B$, also is incorrectly. We now consider the Equation (25) in the above of continuous spectrum of operator $\tilde{H}_{1}$. We have

$$
-\frac{1}{\sqrt{(z-A)^{2}-4 B^{2}}}=-\frac{E}{z-A+2 B} \text {. }
$$

In this equation we find the solutions above of continuous spectrum of operator $\tilde{H}_{1}$. Now we verify the conditions $Z_{i}>A+2 B, i=1,2$. The inequality $z_{1}>A+2 B$, is correctly, and inequality $z_{2}>A+2 B$, is incorrectly. Consequently, in this case the operator $\tilde{H}_{1}$. has a unique eigenvalue $z_{1}=A+\frac{2 B\left(E^{2}+1\right)}{E^{2}-1}$, lying the above of continuous spectrum of operator $\tilde{H}_{1}$.

Let $\varepsilon_{1}=-\frac{2\left(\varepsilon_{2}^{2}+2 B \varepsilon_{2}\right)}{B}$, then the equation of eigenvalues and eigenfunctions take in the form

$$
J(z)=-\frac{E}{z-A-2 B},
$$

where $E=\frac{\left(B+\varepsilon_{2}\right)^{2}}{\varepsilon_{2}^{2}+2 B \varepsilon_{2}}$.

In the below of continuous spectrum of operator $\tilde{H}_{1}$, we have equation of the form

$$
\frac{1}{\sqrt{(A-z)^{2}-4 B^{2}}}=\frac{E}{A-z+2 B} .
$$

From here we find $z_{1}=A-\frac{2 B\left(E^{2}+1\right)}{E^{2}-1}$, and $z_{2}=A+2 B$. The appear inequalities $z_{1}<A-2 B$, is correct, and $z_{2}<A-2 B$, is incorrect. In the above of continuous spectrum of operator $\tilde{H}_{1}$, we have equation of the form

$$
-\frac{1}{\sqrt{(z-A)^{2}-4 B^{2}}}=-\frac{E}{z-A-2 B} \text {. }
$$

It follows that, what $z_{1}=A-\frac{2 B\left(E^{2}+1\right)}{E^{2}-1}$, and $z_{2}=A+2 B$. The inequality $z_{1}>A+2 B$, and $z_{2}>A+2 B$, are incorrectly. Therefore, in this case the operator $\tilde{H}_{1}$ has a unique eigenvalue $z_{1}=A-\frac{2 B\left(E^{2}+1\right)}{E^{2}-1}$, lying the below of continuous spectrum of operator $\tilde{H}_{1}$.

6) If $\varepsilon_{2}>0$ and $\varepsilon_{1}>\frac{2\left(\varepsilon_{2}^{2}+2 B \varepsilon_{2}\right)}{B}$, (respectively, $\varepsilon_{2}<-2 B$ and $\left.\varepsilon_{1}>\frac{2\left(\varepsilon_{2}^{2}+2 B \varepsilon_{2}\right)}{B}\right)$, then consider necessary, that $\varepsilon_{1}=\alpha \times \frac{2\left(\varepsilon_{2}^{2}+2 B \varepsilon_{2}\right)}{B}$, where $\alpha>1$-real number. Then the equation for eigenvalues and eigenfunctions has the form

$$
\left\{\alpha \times \frac{2\left(\varepsilon_{2}^{2}+2 B \varepsilon_{2}\right)}{B} \times B^{2}+\left(\varepsilon_{2}^{2}+2 B \varepsilon_{2}\right)(z-A)\right\} J(z)+\left(B+\varepsilon_{2}\right)^{2}=0,
$$


or

$$
\left(\varepsilon_{2}^{2}+2 B \varepsilon_{2}\right)(z-A+2 \alpha B) J(z)+\left(B+\varepsilon_{2}\right)^{2}=0
$$

From this

$$
J(z)=-\frac{\left(B+\varepsilon_{2}\right)^{2}}{\left(\varepsilon_{2}^{2}+2 B \varepsilon_{2}\right)(z-A+2 \alpha B)} .
$$

We denote $E=\frac{\left(B+\varepsilon_{2}\right)^{2}}{\varepsilon_{2}^{2}+2 B \varepsilon_{2}}$, then

$$
J(z)=-\frac{E}{z-A+2 \alpha B} .
$$

In the first we consider this equation in the below of the continuous spectrum of operator $\tilde{H}_{1}$. Then

$$
\frac{1}{\sqrt{(A-z)^{2}-4 B^{2}}}=\frac{E}{A-z-2 \alpha B} .
$$

This equation has the solutions $z_{1}=A+\frac{2 B\left(\alpha+E \sqrt{E^{2}-1+\alpha^{2}}\right)}{E^{2}-1}$, and $z_{2}=A+\frac{2 B\left(\alpha-E \sqrt{E^{2}-1+\alpha^{2}}\right)}{E^{2}-1}$. Now, we verify the condition $z_{i}<A-2 B, i=1,2$. The solution $z_{1}$ no satisfy the condition $z_{1}<A-2 B$, but $z_{2}$ satisfy the condition $z_{2}<A-2 B$. We now verify the conditions $z_{2}<A-2 \alpha B$. The appear, this inequality is incorrectly. The appear inequalities $z_{1}>A+2 B$ is correct, and $z_{2}>A+2 B$, is incorrect. We now verify the conditions $z_{1}>A-2 \alpha B$. So far as, $A-2 \alpha B<A+2 B$, the appear, this inequality is correctly. Consequently, in this case, the operator $\tilde{H}_{1}$ has a unique eigenvalue $z_{1}=A+\frac{2 B\left(\alpha+E \sqrt{E^{2}-1+\alpha^{2}}\right)}{E^{2}-1}$, above of continuous spectrum of operator $\tilde{H}_{1}$.

7) If $\varepsilon_{2}>0$, and $\varepsilon_{1}<-\frac{2\left(\varepsilon_{2}^{2}+2 B \varepsilon_{2}\right)}{B}$, (respectively, $\varepsilon_{2}<-2 B$, and $\left.\varepsilon_{1}<-\frac{2\left(\varepsilon_{2}^{2}+2 B \varepsilon_{2}\right)}{B}\right)$, then we assume that $\varepsilon_{1}=-\alpha \times \frac{2\left(\varepsilon_{2}^{2}+2 B \varepsilon_{2}\right)}{B}$, where $\alpha>1$-real number. The equation for eigenvalues and eigenfunctions take in the form

$$
\left(\varepsilon_{2}^{2}+2 B \varepsilon_{2}\right)(z-A-2 \alpha B) J(z)=-\left(B+\varepsilon_{2}\right)^{2} .
$$

From here

$$
\begin{aligned}
& \qquad J(z)=-\frac{\left(B+\varepsilon_{2}\right)^{2}}{\left(\varepsilon_{2}^{2}+2 B \varepsilon_{2}\right)(z-A-2 \alpha B)} . \\
& \text { The introduce notation } E=\frac{\left(B+\varepsilon_{2}\right)^{2}}{\varepsilon_{2}^{2}+2 B \varepsilon_{2}} \text {. Then }
\end{aligned}
$$




$$
J(z)=-\frac{E}{z-A-2 \alpha B} .
$$

In the below of the continuous spectrum of operator $\tilde{H}_{1}$, we have the equation

$$
J(z)=\frac{E}{A-z+2 \alpha B},
$$

from here

$$
\frac{1}{\sqrt{(A-z)^{2}-4 B^{2}}}=\frac{E}{A-z+2 \alpha B}
$$

This equation take the form

$$
\left(E^{2}-1\right)(A-z)^{2}-4 \alpha B(A-z)-4 B^{2}\left(E^{2}+\alpha^{2}\right)=0 .
$$

We find $z_{1}=A-\frac{2 B\left(\alpha+E \sqrt{E^{2}-1+\alpha^{2}}\right)}{E^{2}-1}$ and

$z_{2}=A-\frac{2 B\left(\alpha-E \sqrt{E^{2}-1+\alpha^{2}}\right)}{E^{2}-1}$. We now verify the conditions

$z_{i}<m_{1}=A-2 B, i=1,2$. The appear, that $z_{1}<A-2 B$, is correctly and

$Z_{2}<A-2 B$, is incorrectly. Now we consider the Equation (38) in the above of the continuous spectrum of operator $\tilde{H}_{1}$. Then

$$
J(z)=-\frac{E}{z-A-2 \alpha B} .
$$

From this

$$
-\frac{1}{\sqrt{(z-A)^{2}-4 B^{2}}}=-\frac{E}{z-A-2 \alpha B} .
$$

We find $z_{1}=A-\frac{2 B\left(\alpha+E \sqrt{E^{2}-1+\alpha^{2}}\right)}{E^{2}-1}$, and

$z_{2}=A+\frac{2 B\left(-\alpha+E \sqrt{E^{2}-1+\alpha^{2}}\right)}{E^{2}-1}$. We verify the conditions $z_{i}>A+2 B, i=1,2$.

The appear $z_{1}>A+2 B$, it is not true, and the $z_{2}>A+2 B$, is true. We now verify the conditions $z_{2}>A+2 \alpha B$. The appear, this inequality is incorrectly. Consequently, in this case, the operator $\tilde{H}_{1}$ have unique eigenvalue $z_{1}=A-\frac{2 B\left(\alpha+E \sqrt{E^{2}-1+\alpha^{2}}\right)}{E^{2}-1}<m_{1}$, i.e., lying the below of the continuous spectrum of operator $\tilde{H}_{1}$.

8) If $\varepsilon_{2}>0$ and $-\frac{2\left(\varepsilon_{2}^{2}+2 B \varepsilon_{2}\right)}{B}<\varepsilon_{1}<\frac{2\left(\varepsilon_{2}^{2}+2 B \varepsilon_{2}\right)}{B}$ (respectively,

$\varepsilon_{2}<-2 B$ and $\left.-\frac{2\left(\varepsilon_{2}^{2}+2 B \varepsilon_{2}\right)}{B}<\varepsilon_{1}<\frac{2\left(\varepsilon_{2}^{2}+2 B \varepsilon_{2}\right)}{B}\right)$, the we take 
$\varepsilon_{1}=\alpha \times \frac{2\left(\varepsilon_{2}^{2}+2 B \varepsilon_{2}\right)}{B}$, where $-1<\alpha<1-$ real number. Then the equation for eigenvalues and eigenfunctions has the form

$$
\left(\varepsilon_{2}^{2}+2 B \varepsilon_{2}\right)(z-A+2 \alpha B) J(z)=-\left(B+\varepsilon_{2}\right)^{2},|\alpha|<1 .
$$

We denote $E=\frac{\left(B+\varepsilon_{2}\right)^{2}}{\varepsilon_{2}^{2}+2 B \varepsilon_{2}}$. Then the Equation (44) receive the form

$$
J(z)=-\frac{E}{z-A+2 \alpha B} .
$$

In the below of the continuous spectrum of the operator $\tilde{H}_{1}$ we have equation of the form

$$
\frac{1}{\sqrt{(A-z)^{2}-4 B^{2}}}=\frac{E}{A-z-2 \alpha B},|\alpha|<1 .
$$

This equation has a solutions $z_{1}=A+\frac{2 B\left(\alpha+E \sqrt{E^{2}-1+\alpha^{2}}\right)}{E^{2}-1}$, and $z_{2}=A+\frac{2 B\left(\alpha-E \sqrt{E^{2}-1+\alpha^{2}}\right)}{E^{2}-1}$. The inequalities $z_{1}<A-2 B$, and $z_{1}<A-2 \alpha B$, is implements. The inequalities $z_{2}<A-2 B$, is correctly, and the inequality $z_{1}<A-2 B$, is incorrectly. We now verify the conditions $z_{2}<A-2 \alpha B$, since $A-2 B<A-2 \alpha B$, this inequality is true. We now consider the Equation (44) in the above of the continuous spectrum of the operator $\tilde{H}_{1}$. We have the equation of the form

$$
-\frac{1}{\sqrt{(z-A)^{2} 4 B^{2}}}=-\frac{E}{z-A+2 \alpha B} .
$$

This equation has a solutions $z_{1}=A+\frac{2 B\left(\alpha+E \sqrt{E^{2}-1+\alpha^{2}}\right)}{E^{2}-1}$, and $z_{2}=A+\frac{2 B\left(\alpha-E \sqrt{E^{2}-1+\alpha^{2}}\right)}{E^{2}-1}$.

The inequalities $z_{1}>A+2 B$, and $z_{1}>A-2 \alpha B$ is true, as $A+2 B>A-2 \alpha B$, that the inequality $z_{1}>A-2 \alpha B$ is correctly. The inequalities $z_{2}>A+2 B$, and $z_{2}>A+2 \alpha B$ is incorrectly. Consequently, in this case the operator $\tilde{H}_{1}$ has a exactly two eigenvalues $z_{1}=A+\frac{2 B\left(\alpha+E \sqrt{E^{2}-1+\alpha^{2}}\right)}{E^{2}-1}$, and $z_{2}=A+\frac{2 B\left(\alpha-E \sqrt{E^{2}-1+\alpha^{2}}\right)}{E^{2}-1}$, lying the above and below of the continuous spectrum of the operator $\tilde{H}_{1}$.

9) If $-2 B<\varepsilon_{2}<0$, then $\varepsilon_{2}^{2}+2 B \varepsilon_{2}<0$, and the function $\psi(z)=-\frac{\left(B+\varepsilon_{2}\right)^{2}}{\varepsilon_{1} B+\left(\varepsilon_{2}^{2}+2 B \varepsilon_{2}\right)(z-A)}$ is a decreasing function in the intervals 
$\left(-\infty, z_{0}\right)$ and $\left(z_{0},+\infty\right) ;$ By, $z \rightarrow-\infty$ the function $\psi(z) \rightarrow-0$, and by $z \rightarrow z_{0}-0$, the function $\psi(z) \rightarrow-\infty$, and by $z \rightarrow+\infty, \psi(z) \rightarrow+0$, and by $z \rightarrow z_{0}+0, \psi(z) \rightarrow+\infty$. The function $J(z) \rightarrow 0$, by $z \rightarrow-\infty$, and by $z \rightarrow m_{1}-0$, the function $J(z) \rightarrow+\infty$, and by $z \rightarrow M_{1}+0$, the function $J(z) \rightarrow-\infty$, by $z \rightarrow+\infty$, the function $J(z) \rightarrow-0$. Therefore, the equation

$$
\psi(z)=J(z),
$$

that's impossible the solutions in the outside the continuous spectrum of operator $\tilde{H}_{1}$. Therefore, in this case, the operator $\tilde{H}_{1}$ has no eigenvalues lying the outside of the continuous spectrum of the operator $\tilde{H}_{1}$.

Now we consider the two-dimensional case. In two-dimensional case, we have, what the equation $\Delta_{2}(z)=0$, is equivalent to the equation of the form

$$
\left(\varepsilon_{2}+B\right)^{2}+\left\{\varepsilon_{1} B^{2}+\left(\varepsilon_{2}^{2}+2 B \varepsilon_{2}\right)(z-A)\right\} J(z)=0,
$$

where $J(z)=\int_{T^{2}} \frac{d s_{1} \mathrm{~d} s_{2}}{A+2 B\left(\cos s_{1}+\cos s_{2}\right)-z}$. In this case, also $J(z) \rightarrow+0$, as $z \rightarrow-\infty$, and $J(z) \rightarrow+\infty$, as $z \rightarrow m_{2}-0$, and $J(z) \rightarrow-\infty$, as $z \rightarrow M_{2}+0$, and $J(z) \rightarrow-0$, as $z \rightarrow+\infty$. In one- and two-dimensional case the behavior of function $J(z)$ be similarly. Therefore, we have the analogously results, what is find the one-dimensional case.

We consider the three-dimensional case.

Theorem 7. Let $v=3$. Then

1) a) If $\varepsilon_{2}=-B$ and $\varepsilon_{1}<-6 B$ (respectively, $\varepsilon_{2}=-B$ and $\varepsilon_{1}>6 B$,) then the operator $\tilde{H}_{1}$ has a unique eigenvalue $z=A+\varepsilon_{1}$, lying the below (respectively, above) of the continuous spectrum of the operator $\tilde{H}_{1}$.

b) If $\varepsilon_{2}=-B$ and $-6 B \leq \varepsilon_{1}<-2 B$ (respectively, $\varepsilon_{2}=-B$ and $2 B<\varepsilon_{1} \leq 6 B$,) then the operator $\tilde{H}_{1}$ has no eigenvalue, lying the below (respectively, above) of the continuous spectrum of the operator $\tilde{H}_{1}$.

2) If $\varepsilon_{2}=-2 B$ and $\varepsilon_{1}<0, \varepsilon_{1} \leq-\frac{6 B}{W}$, (respectively, $\varepsilon_{2}=-2 B$ and $\varepsilon_{1}>0$, $\varepsilon_{1} \geq \frac{6 B}{W}$ ), then the operator $\tilde{H}_{1}$ has a unique eigenvalue $z_{1}$ (respectively, $z_{2}$ ), lying the below (respectively, above) of the continuous spectrum of the operator $\tilde{H}_{1}$. If $-\frac{6 B}{W} \leq \varepsilon_{1}<0$ (respectively, $0<\varepsilon_{1} \leq \frac{6 B}{W}$ ), then the operator $\tilde{H}_{1}$ has no eigenvalue the outside of the continuous spectrum of operator $\tilde{H}_{1}$.

3) If $\varepsilon_{2}=0$ and $\varepsilon_{1}<0, \varepsilon_{1}<-\frac{6 B}{W}$, (respectively, $\varepsilon_{2}=0$ and $\varepsilon_{1}>0, \varepsilon_{1}>\frac{6 B}{W}$ ), then the operator $\tilde{H}_{1}$ has a unique eigenvalue $z$, lying the below (respectively, above) of the continuous spectrum of the operator $\tilde{H}_{1}$. If $\varepsilon_{2}=0$ and $\varepsilon_{1}<0,-\frac{6 B}{W} \leq \varepsilon_{1}<0$, (respectively, $\varepsilon_{2}=0$ and $0<\varepsilon_{1} \leq \frac{6 B}{W}$ ), then the operator $\tilde{H}_{1}$ has no eigenvalues in the outside of the continuous spectrum of the operator $\tilde{H}_{1}$. 
4) If $\varepsilon_{1}=0$ and $\varepsilon_{2}>0, E>W$, (respectively, $\varepsilon_{1}=0$ and $\varepsilon_{2}<-2 B, E<W$ ), then the operator $\tilde{H}_{1}$ has a unique eigenvalue $z,(\tilde{z})$, where $E=\frac{\left(B+\varepsilon_{2}\right)^{2}}{\varepsilon_{2}^{2}+2 B \varepsilon_{2}}$, lying the below (above) of the continuous spectrum of the operator $\tilde{H}_{1}$. If $\varepsilon_{1}=0$ and $\varepsilon_{2}>0, E<W$, (respectively, $\varepsilon_{1}=0$ and $\varepsilon_{2}<-2 B, E>W$ ), then the operator $\tilde{H}_{1}$ has no eigenvalues the outside the continuous spectrum of the operator $\tilde{H}_{1}$.

5) If $\varepsilon_{1}=\frac{2\left(\varepsilon_{2}^{2}+2 B \varepsilon_{2}\right)}{B}$, (respectively, $\left.\varepsilon_{1}=-\frac{2\left(\varepsilon_{2}^{2}+2 B \varepsilon_{2}\right)}{B}\right)$, then the operator $\tilde{H}_{1}$ has a unique eigenvalue $z$, (respectively, $\tilde{z}$ ), lying the above (respectively, below) of the continuous spectrum of the operator $\tilde{H}_{1}$.

6) If $\varepsilon_{2}>0$ and $\varepsilon_{1}>\frac{2\left(\varepsilon_{2}^{2}+2 B \varepsilon_{2}\right)}{B}$ (respectively, $\varepsilon_{2}<-2 B$ and $\left.\varepsilon_{1}>\frac{2\left(\varepsilon_{2}^{2}+2 B \varepsilon_{2}\right)}{B}\right)$, then the operator $\tilde{H}_{1}$ has a unique eigenvalue $z_{1}$, lying the above of the continuous spectrum of the operator $\tilde{H}_{1}$.

7) If $\varepsilon_{2}>0$ and $\varepsilon_{1}<-\frac{2\left(\varepsilon_{2}^{2}+2 B \varepsilon_{2}\right)}{B}$ (respectively, $\varepsilon_{2}<-2 B$ and $\left.\varepsilon_{1}<-\frac{2\left(\varepsilon_{2}^{2}+2 B \varepsilon_{2}\right)}{B}\right)$, then the operator $\tilde{H}_{1}$ has a unique eigenvalue $z_{1}$, lying the below of the continuous spectrum of the operator $\tilde{H}_{1}$.

8) If $\varepsilon_{2}>0$ and $-\frac{2\left(\varepsilon_{2}^{2}+2 B \varepsilon_{2}\right)}{B}<\varepsilon_{1}<\frac{2\left(\varepsilon_{2}^{2}+2 B \varepsilon_{2}\right)}{B}$ (respectively,

$\varepsilon_{2}<-2 B$ and $\left.-\frac{2\left(\varepsilon_{2}^{2}+2 B \varepsilon_{2}\right)}{B}<\varepsilon_{1}<\frac{2\left(\varepsilon_{2}^{2}+2 B \varepsilon_{2}\right)}{B}\right)$, then the operator $\tilde{H}_{1}$ has a exactly two eigenvalues $Z_{1}$, and $Z_{2}$, lying the above and below of the continuous spectrum of the operator $\tilde{H}_{1}$.

9) If $-2 B<\varepsilon_{2}<0$, then the operator $\tilde{H}_{1}$ has no eigenvalues lying the outside of the continuous spectrum of the operator $\tilde{H}_{1}$.

Proof. In the case $v=3$, the continuous spectrum of the operator $\tilde{H}_{1}$ coincide with segment $\left[m_{3}, M_{3}\right]=[A-6 B, A+6 B]$. Expressing all integrals in the equation

$\Delta_{3}(z)=\left(1+\int_{T^{3}} \frac{\left(\varepsilon_{1}+2 \varepsilon_{2} \sum_{i=1}^{3} \cos s_{i}\right) \mathrm{d} s_{1} \mathrm{~d} s_{2} \mathrm{~d} s_{3}}{A+2 B \sum_{i=1}^{3} \cos s_{i}-z}\right)$
$\times\left(1+6 \varepsilon_{2} \int_{T^{3}} \frac{\cos s_{i} \mathrm{~d} s_{1} \mathrm{~d} s_{2} \mathrm{~d} s_{3}}{A+2 B \sum_{i=1}^{3} \cos s_{i}-z}\right)$
$-6 \varepsilon_{2} \int_{T^{3}} \frac{\mathrm{d} s_{1} \mathrm{~d} s_{2} \mathrm{~d} s_{3}}{A+2 B \sum_{i=1}^{3} \cos s_{i}-z} \int_{T^{3}} \frac{\left(\varepsilon_{1}+2 \varepsilon_{2} \sum_{i=1}^{3} \cos s_{i}\right) \cos s_{1} \mathrm{~d} s_{1} \mathrm{~d} s_{2} \mathrm{~d} s_{3}}{A+2 B \sum_{i=1}^{3} \cos s_{i}-z}=0$ through

the integral $J(z)=\int_{T^{3}} \frac{\mathrm{ds}_{1} \mathrm{ds}_{2} \mathrm{ds}_{3}}{A+2 B \sum_{i=1}^{3} \cos s_{i}-z}$, we find that the equation 
$\Delta_{3}(z)=0$ is equivalent to the equation

$$
\left[\varepsilon_{1} B^{2}+\left(\varepsilon_{2}^{2}+2 B \varepsilon_{2}\right)(z-A)\right] J(z)+\left(B+\varepsilon_{2}\right)^{2}=0 .
$$

Moreover, the function $J(z)=\int_{T^{3}} \frac{\mathrm{d}_{1} \mathrm{~d}_{2} \mathrm{ds}_{3}}{A+2 B \sum_{i=1}^{3} \cos s_{i}-z}$ is a differentiable function on the set $\mathbb{R} \backslash\left[m_{3}, M_{3}\right]$, in addition,

$$
J^{\prime}(z)=\int_{T^{3}} \frac{\mathrm{ds}_{1} \mathrm{ds}_{2} \mathrm{~d} s_{3}}{\left[A+2 B \sum_{i=1}^{3} \cos s_{i}-z\right]^{2}}>0, z \notin\left[m_{3}, M_{3}\right] .
$$

In the three-dimensional case, the integral

$\int_{T^{3}} \frac{d s_{1} d s_{2} d s_{3}}{3+\cos s_{1}+\cos s_{2}+\cos s_{2}}=\int_{T^{3}} \frac{d s_{1} d s_{2} d s_{3}}{3-\cos s_{1}-\cos s_{2}-\cos s_{2}}$ have the finite value, expressing these integral via Watson integral [15]

$$
W=\frac{1}{\pi^{3}} \int_{-\pi}^{\pi} \int_{-\pi}^{\pi} \int_{-\pi}^{\pi} \frac{3 \mathrm{~d} x \mathrm{~d} y \mathrm{~d} z}{3-\cos x-\cos y-\cos z} \approx 1,516 \text {, and taking into account, what }
$$

the measure is normalized, we have, that $J(z)=\frac{W}{6 B}$. Thus the function $J(z)$ is an monotone increasing function on $\left(-\infty, m_{3}\right)$ and on $\left(M_{3},+\infty\right)$. Furthermore, in the three-dimensional case $J(z) \rightarrow+0$ at $z \rightarrow-\infty$, and $J(z)=\frac{W}{6 B}$ as $z=A-6 B$, and $J(z) \rightarrow-0$ as $z \rightarrow+\infty$, and $J(z)=-\frac{W}{6 B}$ as $z=A+6 B$.

If $\varepsilon_{1} B^{2}+\left(\varepsilon_{2}^{2}+2 B \varepsilon_{2}\right)(z-A) \neq 0$ then from (12) follows that

$$
J(z)=-\frac{\left(B+\varepsilon_{2}\right)^{2}}{\varepsilon_{1} B^{2}+\left(\varepsilon_{2}^{2}+2 B \varepsilon_{2}\right)(z-A)} .
$$

The function $\psi(z)=-\frac{\left(B+\varepsilon_{2}\right)^{2}}{\varepsilon_{1} B^{2}+\left(\varepsilon_{2}^{2}+2 B \varepsilon_{2}\right)(z-A)}$ has a point of asymptotic discontinuity $z_{0}=A-\frac{B^{2} \varepsilon_{1}}{\varepsilon_{2}^{2}+2 B \varepsilon_{2}}$. Since $\psi^{\prime}(z)=\frac{\left(B+\varepsilon_{2}\right)^{2}\left(\varepsilon_{2}^{2}+2 B \varepsilon_{2}\right)}{\left[\varepsilon_{1} B^{2}+\left(\varepsilon_{2}^{2}+2 B \varepsilon_{2}\right)(z-A)\right]^{2}}$ for all $z \neq z_{0}$ it follows that the function $\psi(z)$ is an monotone increasing (decreasing) function on $\left(-\infty, z_{0}\right)$ and on $\left(z_{0},+\infty\right)$ in the case $\varepsilon_{2}^{2}+2 B \varepsilon_{2}>0$ (respectively, $\varepsilon_{2}^{2}+2 B \varepsilon_{2}<0$ ), in addition, and if $\varepsilon_{2}>0$, or $\varepsilon_{2}<-2 B$, then $\psi(z) \rightarrow+0$ as $z \rightarrow-\infty, \psi(z) \rightarrow+\infty$ as $z \rightarrow z_{0}-0$, $\psi(z) \rightarrow-\infty$ as

$z \rightarrow z_{0}+0, \psi(z) \rightarrow-0$ as $z \rightarrow+\infty$ (respectively, if $-2 B<\varepsilon_{2}<0$, then $\psi(z) \rightarrow-0$ as $z \rightarrow-\infty, \psi(z) \rightarrow-\infty$ as $z \rightarrow z_{0}-0, \psi(z) \rightarrow+\infty$ as $z \rightarrow z_{0}+0, \psi(z) \rightarrow+0$ as $\left.z \rightarrow+\infty\right)$.

1) If $\varepsilon_{2}=-B$ and $\varepsilon_{1}<-6 B$ (respectively, $\varepsilon_{2}=-B$ and $\varepsilon_{1}>6 B$ ), then the equation for eigenvalues and eigenfunctions (13) has the form (15):

$$
\left\{\varepsilon_{1} B^{2}-B^{2}(z-A)\right\} J(z)=0 .
$$

It is clear, that $J(z) \neq 0$ for the values $z \notin \sigma_{\text {cont }}\left(\tilde{H}_{1}\right)$. Therefore, 
$\varepsilon_{1}-z+A=0$, i.e., $z=A+\varepsilon_{1}$. If $\varepsilon_{1}<-6 B$, then this eigenvalue lying the below of the continuous spectrum of operator $\tilde{H}_{1}$, if $\varepsilon_{1}>6 B$, then this eigenvalue lying the above of the continuous spectrum of operator $\tilde{H}_{1}$. If $-6 B \leq \varepsilon_{1}<-2 B$ (respectively, $2 B<\varepsilon_{1} \leq 6 B$ ), then this eigenvalue not lying in the outside of the continuous spectrum of operator $\tilde{H}_{1}$.

2) If $\varepsilon_{2}=-2 B$ and $\varepsilon_{1}<0$ (respectively, $\varepsilon_{2}=-2 B$ and $\varepsilon_{1}>0$ ), then the equation for the eigenvalues and eigenfunctions has the form $\varepsilon_{1} B^{2} J(z)+B^{2}=0$, that is, $J(z)=-\frac{1}{\varepsilon_{1}}$. In the three-dimensional case $J(z) \rightarrow+0$ as $z \rightarrow-\infty$, and $J(z)=\frac{W}{6 B}$ as $z=A-6 B$, and $J(z) \rightarrow-0$ as $z \rightarrow+\infty$, and $J(z)=-\frac{W}{6 B}$ as $z=A+6 B$. Therefore, in order to the equation $J(z)=-\frac{1}{\varepsilon_{1}}$ in the below (respectively, above) of continuous spectrum of operator $\tilde{H}_{1}$ have the solution, one should implements the inequality $-\frac{1}{\varepsilon_{1}}<\frac{W}{6 B}$, (respectively, $-\frac{1}{\varepsilon_{1}}>-\frac{W}{6 B}$, i.e., $\varepsilon_{1}<-\frac{6 B}{W}, \varepsilon_{1}<0$, (respectively, $\left.\varepsilon_{1}>\frac{6 B}{W}, \varepsilon_{1}>0\right)$. If $-\frac{6 B}{W}<\varepsilon_{1}<0$, (respectively, $0<\varepsilon_{1}<\frac{6 B}{W}$ ), then the operator $\tilde{H}_{1}$ has no eigenvalues the outside the continuous spectrum of operator $\tilde{H}_{1}$.

3) If $\varepsilon_{2}=0$ and $\varepsilon_{1}<0$ (respectively, $\varepsilon_{2}=0$ and $\varepsilon_{1}>0$ ), then the equation for the eigenvalues and eigenfunctions take in the form $\varepsilon_{1} B^{2} J(z)+B^{2}=0$ or $J(z)=-\frac{1}{\varepsilon_{1}}$. In the three-dimensional case $J(z) \rightarrow+0$ as $z \rightarrow-\infty$, and $J(z)=\frac{W}{6 B}$ as $z=A-6 B$, and $\quad J(z) \rightarrow-0$ as $z \rightarrow+\infty$, and $\quad J(z)=-\frac{W}{6 B}$ as $z=A+6 B$. Therefore, in order to the equation $J(z)=-\frac{1}{\varepsilon_{1}}$ in the below (respectively, above) of continuous spectrum of operator $\tilde{H}_{1}$ have the solution, one should implements the inequality $-\frac{1}{\varepsilon_{1}}<\frac{W}{6 B}$, (respectively, $-\frac{1}{\varepsilon_{1}}>-\frac{W}{6 B}$,) i.e., $\varepsilon_{1}<-\frac{6 B}{W}, \varepsilon_{1}<0$, (respectively, $\varepsilon_{1}>\frac{6 B}{W}, \varepsilon_{1}>0$ ). If $-\frac{6 B}{W}<\varepsilon_{1}<0$, (respectively, $0<\varepsilon_{1}<\frac{6 B}{W}$ ), then the operator $\tilde{H}_{1}$ has no eigenvalues the outside the continuous spectrum of operator $\tilde{H}_{1}$.

4) If $\varepsilon_{1}=0$ and $\varepsilon_{2}>0$ (respectively, $\varepsilon_{1}=0$ and $\varepsilon_{2}<-2 B$ ), then the equation for the eigenvalues and eigenfunctions take in the form

$$
\left(\varepsilon_{2}^{2}+2 B \varepsilon_{2}\right)(z-A) J(z)=-\left(B+\varepsilon_{2}\right)^{2},
$$

or

$$
J(z)=-\frac{\left(B+\varepsilon_{2}\right)^{2}}{\left(\varepsilon_{2}^{2}+2 B \varepsilon_{2}\right)(z-A)} .
$$


Denote $E=\frac{\left(B+\varepsilon_{2}\right)^{2}}{\varepsilon_{2}^{2}+2 B \varepsilon_{2}}$. Then $J(z)=-\frac{E}{Z-A}$, or $J(z)=\frac{E}{A-z}$. In the three-dimensional case $J(z) \rightarrow+0$ as $z \rightarrow-\infty$, and $J(z)=\frac{W}{6 B}$ as $z=A-6 B$, and $J(z) \rightarrow-0$ as $z \rightarrow+\infty$, and $J(z)=-\frac{W}{6 B}$ as $z=A+6 B$. Therefore, in order to the equation $J(z)=-\frac{E}{z-A}$ in the below (respectively, above) of continuous spectrum of operator $\tilde{H}_{1}$ have the solution, one should implements the inequality $\frac{E}{6 B}>\frac{W}{6 B}$, (respectively, $\frac{E}{6 B}<\frac{W}{6 B}$,) i.e., $E>W$, (respectively, $E<W$ ). If $\varepsilon_{1}=0$ and $\varepsilon_{2}>0, E<W$, (respectively, $\varepsilon_{1}=0$ and $\left.\varepsilon_{2}<-2 B, E>W\right)$, then the operator $\tilde{H}_{1}$ has no eigenvalues the outside the continuous spectrum of operator $\tilde{H}_{1}$.

5) If $\varepsilon_{1}=\frac{2\left(\varepsilon_{2}^{2}+2 B \varepsilon_{2}\right)}{B}$, then the equation for eigenvalues and eigenfunctions has the form

$$
\left(\varepsilon_{2}^{2}+2 B \varepsilon_{2}\right)(z-A+2 B) J(z)=-\left(B+\varepsilon_{2}\right)^{2},
$$

from this we have equation in the form (13): $J(z)=-\frac{\left(B+\varepsilon_{2}\right)^{2}}{\left(\varepsilon_{2}^{2}+2 B \varepsilon_{2}\right)(z-A+2 B)}$. We denote $E=\frac{\left(B+\varepsilon_{2}\right)^{2}}{\varepsilon_{2}^{2}+2 B \varepsilon_{2}}$. In the first we consider the Equation (14) in the below of continuous spectrum of operator $\tilde{H}_{1}$. In the below of continuous spectrum of operator $\tilde{H}_{1}$, the function $\frac{E}{A-z-2 B} \rightarrow+0$, as $z \rightarrow-\infty$, $\frac{E}{A-z-2 B}=\frac{E}{4 B}$, as $z=A-6 B$, and in the three-dimensional case $J(z) \rightarrow+0$ as $z \rightarrow-\infty$, and $J(z)=\frac{W}{6 B}$ as $z=A-6 B$, and $J(z) \rightarrow-0$ as $z \rightarrow+\infty$, and $J(z)=-\frac{W}{6 B}$ as $z=A+6 B$. Therefore, the below of continuous spectrum of operator $\tilde{H}_{1}$, the equation $J(z)=\frac{E}{A-z-2 B}$ has a unique solution, if $\frac{E}{4 B}>\frac{W}{6 B}$, i.e., $E>\frac{2 W}{3}$. This inequality is incorrect. Therefore, the below of continuous spectrum of operator $\tilde{H}_{1}$, this equation has no solution.

We now consider the equation for eigenvalues and eigenfunctions $J(z)=-\frac{E}{z-A+2 B}$, in the above of continuous spectrum of operator $\tilde{H}_{1}$. In the above of continuous spectrum of operator $\tilde{H}_{1}$, the function $\frac{E}{A-Z-2 B} \rightarrow-0$, as $z \rightarrow+\infty, \frac{E}{A-z-2 B}=-\frac{E}{8 B}$, as $z=A+6 B$, and in the three-dimensional 
case $J(z) \rightarrow-0$ as $z \rightarrow+\infty$, and $J(z)=-\frac{W}{6 B}$ as $z=A+6 B$. Therefore the above of continuous spectrum of operator $\tilde{H}_{1}$, the equation $J(z)=\frac{E}{A-z-2 B}$ has a unique solution, if $-\frac{E}{8 B}>-\frac{W}{6 B}$, i.e., $E<\frac{4 W}{3}$. This inequality correctly. Therefore, the above of continuous spectrum of operator $\tilde{H}_{1}$, this equation has a unique solution $z$.

If $\varepsilon_{1}=-\frac{2\left(\varepsilon_{2}^{2}+2 B \varepsilon_{2}\right)}{B}$, then the equation for eigenvalues and eigenfunctions has the form

$$
\left(\varepsilon_{2}^{2}+2 B \varepsilon_{2}\right)(z-A-2 B) J(z)=-\left(B+\varepsilon_{2}\right)^{2},
$$

from this we have the equation in the form (14):

$J(z)=-\frac{\left(B+\varepsilon_{2}\right)^{2}}{\left(\varepsilon_{2}^{2}+2 B \varepsilon_{2}\right)(z-A+2 B)}$. We denote $E=\frac{\left(B+\varepsilon_{2}\right)^{2}}{\varepsilon_{2}^{2}+2 B \varepsilon_{2}}$. In the first we consider the Equation (14) in the below of continuous spectrum of operator $\tilde{H}_{1}$. In the below of continuous spectrum of operator $\tilde{H}_{1}$, the function $\frac{E}{A-z+2 B} \rightarrow+0$, as $z \rightarrow-\infty, \frac{E}{A-z+2 B}=\frac{E}{8 B}$, as $z=A-6 B$, and in the three-dimensional case $J(z) \rightarrow+0$ as $z \rightarrow-\infty$, and $J(z)=\frac{W}{6 B}$ as $z=A-6 B$, and $J(z) \rightarrow-0$ as $z \rightarrow+\infty$, and $J(z)=-\frac{W}{6 B}$ as $z=A+6 B$. Therefore, the below of continuous spectrum of operator $\tilde{H}_{1}$, the equation $J(z)=\frac{E}{A-z+2 B}$ has a unique solution, if $\frac{E}{8 B}<\frac{W}{6 B}$, i.e., $E<\frac{4 W}{3}$. This inequality is correct. Therefore, the below of continuous spectrum of operator $\tilde{H}_{1}$, this equation has a unique solution.

We now consider the equation for eigenvalues and eigenfunctions $J(z)=-\frac{E}{z-A-2 B}$, in the above of continuous spectrum of operator $\tilde{H}_{1}$. In the above of continuous spectrum of operator $\tilde{H}_{1}$, the function $\frac{E}{A-Z+2 B} \rightarrow-0$, as $z \rightarrow+\infty, \frac{E}{A-z+2 B}=-\frac{E}{4 B}$, as $z=A+6 B$, and in the three-dimensional case $J(z) \rightarrow-0$ as $z \rightarrow+\infty$, and $J(z)=-\frac{W}{6 B}$ as $z=A+6 B$. Therefore, the above of continuous spectrum of operator $\tilde{H}_{1}$, the equation $J(z)=\frac{E}{A-z+2 B}$ has a unique solution, if $-\frac{E}{4 B}>-\frac{W}{6 B}$, i.e., $E<\frac{2 W}{3}$. This inequality is incorrect. Therefore, the above of continuous spectrum of operator $\tilde{H}_{1}$, this equation has no solution.

6) If $\varepsilon_{2}>0$ and $\varepsilon_{1}>\frac{2\left(\varepsilon_{2}^{2}+2 B \varepsilon_{2}\right)}{B}$, (respectively, $\varepsilon_{2}<-2 B$ and 
$\left.\varepsilon_{1}>\frac{2\left(\varepsilon_{2}^{2}+2 B \varepsilon_{2}\right)}{B}\right)$, then consider necessary, that $\varepsilon_{1}=\alpha \times \frac{2\left(\varepsilon_{2}^{2}+2 B \varepsilon_{2}\right)}{B}$, where $\alpha>1-$ real number. Then the equation for eigenvalues and eigenfunctions has the form

$$
\left\{\alpha \times \frac{2\left(\varepsilon_{2}^{2}+2 B \varepsilon_{2}\right)}{B} \times B^{2}+\left(\varepsilon_{2}^{2}+2 B \varepsilon_{2}\right)(z-A)\right\} J(z)+\left(B+\varepsilon_{2}\right)^{2}=0,
$$

or

$$
\left(\varepsilon_{2}^{2}+2 B \varepsilon_{2}\right)(z-A+2 \alpha B) J(z)+\left(B+\varepsilon_{2}\right)^{2}=0 .
$$

From this $J(z)=-\frac{\left(B+\varepsilon_{2}\right)^{2}}{\left(\varepsilon_{2}^{2}+2 B \varepsilon_{2}\right)(z-A+2 \alpha B)}$. We denote $E=\frac{\left(B+\varepsilon_{2}\right)^{2}}{\varepsilon_{2}^{2}+2 B \varepsilon_{2}}$, then $J(z)=-\frac{E}{z-A+2 \alpha B}$. In the first we consider this equation in the below of the continuous spectrum of operator $\tilde{H}_{1}$. Then $J(z) \rightarrow+0$, as $z \rightarrow-\infty$, $J(z)=\frac{W}{6 B}$, as $z=A-6 B,-\frac{E}{z-A+2 \alpha B} \rightarrow+0$, as $z \rightarrow-\infty$, and $-\frac{E}{z-A+2 \alpha B}=\frac{E}{(6-2 \alpha) B}$, as $z=A-6 B$. The equation

$$
J(z)=-\frac{E}{z-A+2 \alpha B}
$$

has a unique solution, if $\frac{E}{(6-2 \alpha) B}<\frac{W}{6 B}$. From here $E<\frac{(3-\alpha) W}{3}$. This inequality is incorrect. Therefore, the below of continuous spectrum of operator $\tilde{H}_{1}$, the operator $\tilde{H}_{1}$ has no eigenvalues.

The above of continuous spectrum of operator $\tilde{H}_{1}$, we have the $J(z) \rightarrow-0$, if $z \rightarrow+\infty, J(z)=-\frac{W}{6 B}$, if $z=A-6 B$. Besides, $-\frac{E}{z-A+2 \alpha B} \rightarrow-0$, as $z \rightarrow+\infty,-\frac{E}{z-A+2 \alpha B}=-\frac{E}{6 B+2 \alpha B}$, if $z=A+6 B$.

The equation

$$
J(z)=-\frac{E}{z-A+2 \alpha B}
$$

have a unique solution, if $-\frac{E}{(6+2 \alpha) B}>-\frac{W}{6 B}$. From here $E<\frac{(3+\alpha) W}{3}$. This inequality is correctly. Therefore, the above of continuous spectrum of operator $\tilde{H}_{1}$, the operator $\tilde{H}_{1}$ has a unique eigenvalues $z_{1}$.

7) If $\varepsilon_{2}>0$, and $\varepsilon_{1}<-\frac{2\left(\varepsilon_{2}^{2}+2 B \varepsilon_{2}\right)}{B}$, (respectively, $\varepsilon_{2}<-2 B$, and $\left.\varepsilon_{1}<-\frac{2\left(\varepsilon_{2}^{2}+2 B \varepsilon_{2}\right)}{B}\right)$, then we assume that $\varepsilon_{1}=-\alpha \times \frac{2\left(\varepsilon_{2}^{2}+2 B \varepsilon_{2}\right)}{B}$, where 
$\alpha>1$-real number. The equation for eigenvalues and eigenfunctions take in the form

$$
\left(\varepsilon_{2}^{2}+2 B \varepsilon_{2}\right)(z-A-2 \alpha B) J(z)=-\left(B+\varepsilon_{2}\right)^{2} .
$$

From here

$$
J(z)=-\frac{\left(B+\varepsilon_{2}\right)^{2}}{\left(\varepsilon_{2}^{2}+2 B \varepsilon_{2}\right)(z-A-2 \alpha B)} .
$$

The introduce notation $E=\frac{\left(B+\varepsilon_{2}\right)^{2}}{\varepsilon_{2}^{2}+2 B \varepsilon_{2}}$. Then we have the equation in the form (15): $J(z)=-\frac{E}{z-A-2 \alpha B}$. In the below of the continuous spectrum of operator $\tilde{H}_{1}$, we have the equation $J(z)=\frac{E}{A-z+2 \alpha B}$. In the below of continuous spectrum of operator $\tilde{H}_{1},-\frac{E}{z-A-2 \alpha B} \rightarrow+0$, as $z \rightarrow-\infty$, $-\frac{E}{z-A-2 \alpha B}=\frac{E}{6 B+2 \alpha B}$, as $z=A-6 B$.

The equation $J(z)=-\frac{E}{z-A+2 \alpha B}$ have a unique solution, if $\frac{E}{(6+2 \alpha) B}<\frac{W}{6 B}$. From here $E<\frac{(3+\alpha) W}{3}$. This inequality is correctly. Therefore, the below of continuous spectrum of operator $\tilde{H}_{1}$, the operator $\tilde{H}_{1}$ has a unique eigenvalues.

In the above of continuous spectrum of operator $\tilde{H}_{1},-\frac{E}{z-A-2 \alpha B} \rightarrow-0$, as $z \rightarrow-\infty,-\frac{E}{z-A-2 \alpha B}=-\frac{E}{6 B-2 \alpha B}$, as $z=A+6 B$. Therefore, the above of continuous spectrum of operator $\tilde{H}_{1}$, the operator $\tilde{H}_{1}$ has a unique eigenvalues, if $-\frac{E}{6 B-2 \alpha B}=-\frac{W}{6 B}$. From here $E<\frac{(3-\alpha) W}{3}$, what is incorrectly. Therefore, the above of continuous spectrum of operator $\tilde{H}_{1}$, the operator $\tilde{H}_{1}$ has no eigenvalues.

8) If $\varepsilon_{2}>0$ and $-\frac{2\left(\varepsilon_{2}^{2}+2 B \varepsilon_{2}\right)}{B}<\varepsilon_{1}<\frac{2\left(\varepsilon_{2}^{2}+2 B \varepsilon_{2}\right)}{B}$ (respectively, $\varepsilon_{2}<-2 B$ and $\left.-\frac{2\left(\varepsilon_{2}^{2}+2 B \varepsilon_{2}\right)}{B}<\varepsilon_{1}<\frac{2\left(\varepsilon_{2}^{2}+2 B \varepsilon_{2}\right)}{B}\right)$, the we take $\varepsilon_{1}=\alpha \times \frac{2\left(\varepsilon_{2}^{2}+2 B \varepsilon_{2}\right)}{B}$, where $-1<\alpha<1-$ real number. Then the equation for eigenvalues and eigenfunctions has the form:

$$
\left(\varepsilon_{2}^{2}+2 B \varepsilon_{2}\right)(z-A+2 \alpha B) J(z)=-\left(B+\varepsilon_{2}\right)^{2},|\alpha|<1 .
$$

We denote $E=\frac{\left(B+\varepsilon_{2}\right)^{2}}{\varepsilon_{2}^{2}+2 B \varepsilon_{2}}$. Then the Equation (61) receive the form 


$$
J(z)=-\frac{E}{z-A+2 \alpha B} .
$$

In the below of continuous spectrum of operator $\tilde{H}_{1}$, we have

$$
-\frac{E}{z-A+2 \alpha B} \rightarrow+0 \text {, as } z \rightarrow-\infty \text {, and }-\frac{E}{z-A+2 \alpha B}=\frac{E}{2 B(3-\alpha)} \text {, as }
$$

$z=A-6 B$. The equation $J(z)=-\frac{E}{z-A+2 \alpha B}$ have a unique solution the below of continuous spectrum of operator $\tilde{H}_{1}$, if $\frac{E}{(6-2 \alpha) B}>\frac{W}{6 B}$. From here $E>\frac{(3-\alpha) W}{3}$. This inequality is correctly. Therefore, the below of continuous spectrum of operator $\tilde{H}_{1}$, the operator $\tilde{H}_{1}$ has a unique eigenvalues $z_{1}$.

The above of of continuous spectrum of operator $\tilde{H}_{1}$, we have $-\frac{E}{z-A+2 \alpha B} \rightarrow-0$, as $z \rightarrow+\infty$, and $-\frac{E}{z-A+2 \alpha B}=-\frac{E}{2 B(3+\alpha)}$, as $z=A+6 B$. The equation $J(z)=-\frac{E}{z-A+2 \alpha B}$ have a unique solution the above of $\tilde{H}_{1}$ if $-\frac{E}{2 B(3+\alpha)}>-\frac{W}{6 B}$, i.e., $E<\frac{(3+\alpha) W}{3}$. This inequality is correct.

Consequently, in this case the operator $\tilde{H}_{1}$ have two eigenvalues $z_{1}$ and $z_{2}$, lying the below and above of continuous spectrum of operator $\tilde{H}_{1}$.

9) If $-2 B<\varepsilon_{2}<0$, then $\varepsilon_{2}^{2}+2 B \varepsilon_{2}<0$, and the function $\psi(z)=-\frac{\left(B+\varepsilon_{2}\right)^{2}}{\varepsilon_{1} B+\left(\varepsilon_{2}^{2}+2 B \varepsilon_{2}\right)(z-A)}$ is a decreasing function in the intervals $\left(-\infty, z_{0}\right)$ and $\left(z_{0},+\infty\right)$; By, $z \rightarrow-\infty$ the function $\psi(z) \rightarrow-0$, and by $z \rightarrow z_{0}-0$, the function $\psi(z) \rightarrow-\infty$, and by $z \rightarrow+\infty, \psi(z) \rightarrow+0$, and by $z \rightarrow z_{0}+0, \psi(z) \rightarrow+\infty$. The function $J(z) \rightarrow+0$, by $z \rightarrow-\infty$, and by $z=A-6 B$, the function $J(z)=\frac{W}{6 B}$, and by $z=A+6 B$, the function $J(z)=-\frac{W}{6 B}$, by $z \rightarrow+\infty$, the function $J(z) \rightarrow-0$. Therefore, the equation $\psi(z)=J(z)$, that's impossible the solutions in the outside the continuous spectrum of operator $\tilde{H}_{1}$. Therefore, in this case, the operator $\tilde{H}_{1}$ has no eigenvalues lying the outside of the continuous spectrum of the operator $\tilde{H}_{1}$.

From obtaining results is obviously, that the spectrum of operator $\tilde{H}_{1}$ is consists from continuous spectrum and no more than two eigenvalues.

Using tensor products of Hilbert spaces and Using tensor products of operators in Hilbert spaces [16], we can verify that the operator $\tilde{H}_{3 / 2}^{q}$ can be represented in the form

$$
\tilde{H}_{3 / 2}^{q}=\tilde{H}_{1} \otimes I \otimes I+I \otimes \tilde{H}_{1} \otimes I+I \otimes I \otimes \tilde{H}_{1},
$$

where $I$ is the unit operator in the space $\tilde{\mathscr{C}}_{1}$.

The spectrum of the operator $A \otimes I+I \otimes B$, where $A$ and $B$ are densely de- 
fined bounded linear operators, was studied in [17] [18] [19]. In this work explicit formulas were given there that express the essential spectrum $\sigma_{\text {ess }}(A \otimes I+I \otimes B)$ of $A \otimes I+I \otimes B$ and the discrete spectrum $\sigma_{\text {disc }}(A \otimes I+I \otimes B)$ in terms of the spectrum $\sigma(A)$ of $A$ and the discrete spectrum $\sigma_{\text {disc }}(A)$ of $A$ and in terms of the spectrum $\sigma(B)$ of $B$ and the discrete spectrum $\sigma_{\text {disc }}(B)$ of $B$ :

$$
\begin{aligned}
\sigma_{\text {disc }}(A \otimes I+I \otimes B)= & \left\{\sigma(A) \backslash \sigma_{\text {ess }}(A)+\sigma(B) \backslash \sigma_{\text {ess }}(B)\right\} \backslash\left\{\left(\sigma_{\text {ess }}(A)\right.\right. \\
& \left.+\sigma(B)) \cup\left(\sigma(A)+\sigma_{\text {ess }}(B)\right)\right\}, \\
\sigma_{\text {ess }}(A \otimes I+I \otimes B) & =\left(\sigma_{\text {ess }}(A)+\sigma(B)\right) \bigcup\left(\sigma(A)+\sigma_{\text {ess }}(B)\right) .
\end{aligned}
$$

It is clear that $\sigma(A \otimes I+I \otimes B)=\{\lambda+\mu: \lambda \in \sigma(A), \mu \in \sigma(B)\}$.

\section{Structure of the Essential Spectrum and Discrete Spectrum of Operator $\tilde{\boldsymbol{H}}_{3 / 2}^{q}$}

Now, using the obtained results and representation (63), and Formulas (64), (65), we describe the structure of the essential spectrum and the discrete spectrum of the operator $\tilde{H}_{3 / 2}^{q}$.

The next theorems is described the structure of the essential spectrum and the discrete spectrum of the operator $\tilde{H}_{3 / 2}^{q}$ :

Theorem 8. Let $v=1$. Then

1) If $\varepsilon_{2}=-B$ and $\varepsilon_{1}<-2 B$ (respectively, $\varepsilon_{2}=-B$ and $\varepsilon_{1}>2 B$,) then the essential spectrum of the operator $\tilde{H}_{3 / 2}^{q}$ is consists of the union of three segments

$$
\begin{aligned}
\sigma_{\text {ess }}\left(\tilde{H}_{3 / 2}^{q}\right)= & {[3 A-6 B, 3 A+6 B] \cup[2 A-4 B+z, 2 A+4 B+z] \text { and dis- } } \\
& \cup[A-2 B+2 z, A+2 B+2 z]
\end{aligned}
$$

crete spectrum of the operator $\tilde{H}_{3 / 2}^{q}$ is consists of a single eigenvalue, $\sigma_{\text {disc }}\left(\tilde{H}_{3 / 2}^{q}\right)=\{3 z\}$, where $z=A+\varepsilon_{1}$.

2) If $\varepsilon_{2}=-2 B$ and $\varepsilon_{1}<0$ (respectively, $\varepsilon_{2}=-2 B$ and $\varepsilon_{1}>0$ ), then the essential spectrum of the operator $\tilde{H}_{3 / 2}^{q}$ is consists of the union of three segments: $\sigma_{\text {ess }}\left(\tilde{H}_{3 / 2}^{q}\right)=[3 A-6 B, 3 A+6 B] \cup[2 A-4 B+z, 2 A+4 B+z]$, and dis-

$$
\cup[A-2 B+2 z, A+2 B+2 z]
$$

crete spectrum of the operator $\tilde{H}_{3 / 2}^{q}$ is consists of a single eigenvalue: $\sigma_{\text {disc }}\left(\tilde{H}_{3 / 2}^{q}\right)=\{3 z\}$, where $z=A-\sqrt{4 B^{2}+\varepsilon_{1}^{2}}$ (respectively, $z=A+\sqrt{4 B^{2}+\varepsilon_{1}^{2}}$ ).

3) If $\varepsilon_{2}=0$ and $\varepsilon_{1}<0$ (respectively, $\varepsilon_{2}=0$ and $\varepsilon_{1}>0$ ), then the essential spectrum of the operator $\tilde{H}_{3 / 2}^{q}$ is consists of the union of three segments: $\begin{aligned} \sigma_{e s s}\left(\tilde{H}_{3 / 2}^{q}\right)= & {[3 A-6 B, 3 A+6 B] \cup[2 A-4 B+z, 2 A+4 B+z], \text { and discrete spec- } } \\ & \bigcup[A-2 B+2 z, A+2 B+2 z]\end{aligned}$ trum of the operator $\tilde{H}_{3 / 2}^{q}$ is consists of a single eigenvalue: $\sigma_{\text {disc }}\left(\tilde{H}_{3 / 2}^{q}\right)=\{3 z\}$, where $\mathrm{z}=A-\sqrt{4 B^{2}+\varepsilon_{1}^{2}}$ (respectively, $\mathrm{z}=A+\sqrt{4 B^{2}+\varepsilon_{1}^{2}}$ ).

4) If $\varepsilon_{1}=0$ and $\varepsilon_{2}>0$ (respectively, $\varepsilon_{1}=0$ and $\varepsilon_{2}<-2 B$ ), then the es- 
sential spectrum of the operator $\tilde{H}_{3 / 2}^{q}$ is consists of the union of three segments: $\begin{aligned} \sigma_{\text {ess }}\left(\tilde{H}_{3 / 2}^{q}\right)= & {[3 A-6 B, 3 A+6 B] \cup[2 A-4 B+z, 2 A+4 B+z], \text { and discrete spec- } } \\ & \bigcup[A-2 B+2 z, A+2 B+2 z],\end{aligned}$ trum of the operator $\tilde{H}_{3 / 2}^{q}$ is consists of a single eigenvalue: $\sigma_{\text {disc }}\left(\tilde{H}_{3 / 2}^{q}\right)=\{3 z\}$, where $z=A-\frac{2 B E}{\sqrt{E^{2}-1}}$, (respectively, $\left(z=A+\frac{2 B E}{\sqrt{E^{2}-1}}\right)$, and $E=\frac{\left(B+\varepsilon_{2}\right)^{2}}{\varepsilon_{2}^{2}+2 B \varepsilon_{2}}$. 5) If $\varepsilon_{1}=\frac{2\left(\varepsilon_{2}^{2}+2 B \varepsilon_{2}\right)}{B}$, (respectively, $\left.\varepsilon_{1}=-\frac{2\left(\varepsilon_{2}^{2}+2 B \varepsilon_{2}\right)}{B}\right)$, then the essential spectrum of the operator $\tilde{H}_{3 / 2}^{q}$ is consists of the union of three segments: $\begin{aligned} \sigma_{\text {ess }}\left(\tilde{H}_{3 / 2}^{q}\right)= & {[3 A-6 B, 3 A+6 B] \cup[2 A-4 B+z, 2 A+4 B+z], \text { and discrete spec- } } \\ & \bigcup[A-2 B+2 z, A+2 B+2 z]\end{aligned}$ trum of the operator $\tilde{H}_{3 / 2}^{q}$ is consists of a single eigenvalue: $\sigma_{\text {disc }}\left(\tilde{H}_{3 / 2}^{q}\right)=\{3 z\}$, where $z=A+\frac{2 B\left(E^{2}+1\right)}{E^{2}-1}$, (respectively, $\left.z=A-\frac{2 B\left(E^{2}+1\right)}{E^{2}-1}\right)$, and $E=\frac{\left(B+\varepsilon_{2}\right)^{2}}{\varepsilon_{2}^{2}+2 B \varepsilon_{2}}$.

6) If $\varepsilon_{2}>0$ and $\varepsilon_{1}>\frac{2\left(\varepsilon_{2}^{2}+2 B \varepsilon_{2}\right)}{B}$ (respectively, $\varepsilon_{2}<-2 B$ and $\left.\varepsilon_{1}>\frac{2\left(\varepsilon_{2}^{2}+2 B \varepsilon_{2}\right)}{B}\right)$, then the essential spectrum of the operator $\tilde{H}_{3 / 2}^{q}$ is consists of the union of three segments:

$$
\begin{aligned}
\sigma_{\text {ess }}\left(\tilde{H}_{3 / 2}^{q}\right)= & {[3 A-6 B, 3 A+6 B] \cup[2 A-4 B+z, 2 A+4 B+z], \text { and discrete spec- } } \\
& \cup[A-2 B+2 z, A+2 B+2 z]
\end{aligned}
$$

trum of the operator $\tilde{H}_{3 / 2}^{q}$ is consists of a single eigenvalue: $\sigma_{\text {disc }}\left(\tilde{H}_{3 / 2}^{q}\right)=\{3 z\}$, where $z=A+\frac{2 B\left(\alpha+E \sqrt{E^{2}-1+\alpha^{2}}\right)}{E^{2}-1}$, and $E=\frac{\left(B+\varepsilon_{2}\right)^{2}}{\varepsilon_{2}^{2}+2 B \varepsilon_{2}}$, and the real number $\alpha>1$;

7) If $\varepsilon_{2}>0$ and $\varepsilon_{1}<-\frac{2\left(\varepsilon_{2}^{2}+2 B \varepsilon_{2}\right)}{B}$ (respectively, $\varepsilon_{2}<-2 B$ and $\left.\varepsilon_{1}<-\frac{2\left(\varepsilon_{2}^{2}+2 B \varepsilon_{2}\right)}{B}\right)$, then the essential spectrum of the operator $\tilde{H}_{3 / 2}^{q}$ is consists of the union of three segments:

$$
\begin{aligned}
\sigma_{\text {ess }}\left(\tilde{H}_{3 / 2}^{q}\right)= & {[3 A-6 B, 3 A+6 B] \cup[2 A-4 B+z, 2 A+4 B+z], \text { and discrete spec- } } \\
& \cup[A-2 B+2 z, A+2 B+2 z]
\end{aligned}
$$

trum of the operator $\tilde{H}_{3 / 2}^{q}$ is consists of a single eigenvalue: $\sigma_{\text {disc }}\left(\tilde{H}_{3 / 2}^{q}\right)=\{3 z\}$, where $z=A-\frac{2 B\left(\alpha+E \sqrt{E^{2}-1+\alpha^{2}}\right)}{E^{2}-1}$, and $E=\frac{\left(B+\varepsilon_{2}\right)^{2}}{\varepsilon_{2}^{2}+2 B \varepsilon_{2}}$, and the real number $\alpha>1$. 
8) If $\varepsilon_{2}>0$ and $-\frac{2\left(\varepsilon_{2}^{2}+2 B \varepsilon_{2}\right)}{B}<\varepsilon_{1}<\frac{2\left(\varepsilon_{2}^{2}+2 B \varepsilon_{2}\right)}{B}$ (respectively,

$\varepsilon_{2}<-2 B$ and $\left.-\frac{2\left(\varepsilon_{2}^{2}+2 B \varepsilon_{2}\right)}{B}<\varepsilon_{1}<\frac{2\left(\varepsilon_{2}^{2}+2 B \varepsilon_{2}\right)}{B}\right)$, then the essential spec-

trum of the operator $\tilde{H}_{3 / 2}^{q}$ is consists of the union of six segments:

$\sigma_{\text {ess }}\left(\tilde{H}_{3 / 2}^{q}\right)=[3 A-6 B, 3 A+6 B] \cup\left[2 A-4 B+z_{1}, 2 A+4 B+z_{1}\right]$

$\cup\left[2 A-4 B+z_{2}, 2 A+4 B+z_{2}\right] \cup\left[A-2 B+2 z_{1}, A+2 B+2 z_{1}\right] \quad$, and discrete

$\bigcup\left[A-2 B+z_{1}+z_{2}, A+2 B+z_{1}+z_{2}\right] \cup\left[A-2 B+2 z_{2}, A+2 B+z_{2}\right]$

spectrum of the operator $\tilde{H}_{3 / 2}^{q}$ is consists of a four eigenvalues:

$\sigma_{\text {disc }}\left(\tilde{H}_{3 / 2}^{q}\right)=\left\{3 z_{1}, 2 z_{1}+z_{2}, z_{1}+2 z_{2}, 3 z_{2}\right\}$, where

$z_{1}=A+\frac{2 B\left(\alpha+E \sqrt{E^{2}-1+\alpha^{2}}\right)}{E^{2}-1}$, and $z_{2}=A+\frac{2 B\left(\alpha-E \sqrt{E^{2}-1+\alpha^{2}}\right)}{E^{2}-1}$, and

$E=\frac{\left(B+\varepsilon_{2}\right)^{2}}{\varepsilon_{2}^{2}+2 B \varepsilon_{2}}$, and the real number $|\alpha|<1$.

9) If $-2 B<\varepsilon_{2}<0$, then the essential spectrum of the operator $\tilde{H}_{3 / 2}^{q}$ is consists is a single segment $\sigma_{\text {ess }}\left(\tilde{H}_{3 / 2}^{q}\right)=[3 A-6 B, 3 A+6 B]$, and discrete spectrum of the operator $\tilde{H}_{3 / 2}^{q}$ is empty.

Proof. 1) It follows from representation (63) and from Formulas (64) and (65), and from Theorem 6, that in one-dimensional case, the continuous spectrum of the operator $\tilde{H}_{1}$ is consists from $\sigma_{\text {cont }}\left(\tilde{H}_{1}\right)=[A-2 B, A+2 B]$, and discrete spectrum of the operator $\tilde{H}_{1}$ is consists of unique eigenvalue $z$. Therefore, the essential spectrum of the operator $\tilde{H}_{1} \otimes I \otimes I+I \otimes \tilde{H}_{1} \otimes I+I \otimes I \otimes \tilde{H}_{1}$ is consists from of the union of three segments $[3 A-6 B, 3 A+6 B]$ and $[2 A-4 B+z, 2 A+4 B+z]$, and $[A-2 B+2 z, A+2 B+2 z]$, and discrete spectrum of the operator $\tilde{H}_{1} \otimes I \otimes I+I \otimes \tilde{H}_{1} \otimes I+I \otimes I \otimes \tilde{H}_{1}$ is consists is the unique eigenvalue $3 z$. These are given to the proof of statement 1 ) from Theorem 8.

The statements (2)-(7) from Theorem 8 are proved similarly.

We now are proving the statement 8) from Theorem 8. It can be seen from Theorem 6 (statement 8 ) in one-dimensional case the operator $\tilde{H}_{1}$ has exactly two eigenvalues $z_{1}$ and $z_{2}$ outside the domain of the continuous spectrum of operator $\tilde{H}_{1}$. Therefore, the set $\sigma_{\text {ess }}\left(\tilde{H}_{3 / 2}^{q}\right)$ consists of the union of six intervals $[3 A-6 B, 3 A+6 B],\left[2 A-4 B+z_{1}, 2 A+4 B+z_{1}\right]$ and

$\left[2 A-4 B+z_{2}, 2 A+4 B+z_{2}\right]$ and $\left[A-2 B+2 z_{1}, A+2 B+2 z_{1}\right]$, and

$\left[A-2 B+z_{1}+z_{2}, A+2 B+z_{1}+z_{2}\right]$, and $\left[A-2 B+2 z_{2}, A+2 B+2 z_{2}\right]$, i.e.,

$\sigma_{\text {ess }}\left(\tilde{H}_{3 / 2}^{q}\right)=[3 A-6 B, 3 A+6 B] \cup\left[2 A-4 B+z_{1}, 2 A+4 B+z_{1}\right]$

$\cup\left[2 A-4 B+z_{2}, 2 A+4 B+z_{2}\right] \cup\left[A-2 B+2 z_{1}, A+2 B+2 z_{1}\right]$. The numbers

$\bigcup\left[A-2 B+z_{1}+z_{2}, A+2 B+z_{1}+z_{2}\right] \cup\left[A-2 B+2 z_{2}, A+2 B+2 z_{2}\right]$

$3 z_{1}, 3 z_{2}$, and $2 z_{1}+z_{2}$, and $z_{1}+2 z_{2}$, are eigenvalues of operator

$\tilde{H}_{1} \otimes I \otimes I+I \otimes \tilde{H}_{1} \otimes I+I \otimes I \otimes \tilde{H}_{1}$. These is given to the proof of statement 8) from Theorem 8 . 
We now are proving the statement 9) from Theorem 8. It can be seen from Theorem 6 (statement 9) in one-dimensional case the operator $\tilde{H}_{1}$ has no eigenvalues the outside the continuous spectrum of operator $\tilde{H}_{1}$. Therefore, the set $\sigma_{\text {ess }}\left(\tilde{H}_{3 / 2}^{q}\right)$ consists of a single segment: $\sigma_{\text {ess }}\left(\tilde{H}_{3 / 2}^{q}\right)=[3 A-6 B, 3 A+6 B]$, and the operator also has no eigenvalues, i.e.,

$\sigma_{\text {disc }}\left(\tilde{H}_{1} \otimes I \otimes I+I \otimes \tilde{H}_{1} \otimes I+I \otimes I \otimes \tilde{H}_{1}\right)=\varnothing$.

The next theorems described the structure of essential spectrum of the operator $\tilde{H}_{3 / 2}^{q}$ in a three-dimensional case.

Theorem 9. Let $v=3$. Then

1) a) If $\varepsilon_{2}=-B$ and $\varepsilon_{1}<-6 B$ (respectively, $\varepsilon_{2}=-B$ and $\varepsilon_{1}>6 B$ ), then the essential spectrum of the operator $\tilde{H}_{3 / 2}^{q}$ is consists of the union of three segments $\sigma_{\text {ess }}\left(\tilde{H}_{3 / 2}^{q}\right)=[3 A-18 B, 3 A+18 B] \cup[2 A-12 B+z, 2 A+12 B+z]$,

$$
\cup[A-6 B+2 z, A+6 B+2 z]
$$

and discrete spectrum of the operator $\tilde{H}_{3 / 2}^{q}$ is consists of a single eigenvalue, $\sigma_{\text {disc }}\left(\tilde{H}_{3 / 2}^{q}\right)=\{3 z\}$, where $z=A+\varepsilon_{1}$.

b) If $\varepsilon_{2}=-B$ and $-6 B \leq \varepsilon_{1}<-2 B$ (respectively, $\varepsilon_{2}=-B$ and $2 B<\varepsilon_{1} \leq 6 B$,) then the essential spectrum of the operator $\tilde{H}_{3 / 2}^{q}$ is consists of a single segment $\sigma_{\text {ess }}\left(\tilde{H}_{3 / 2}^{q}\right)=[3 A-18 B, 3 A+18 B]$, and discrete spectrum of the operator $\tilde{H}_{3 / 2}^{q}$ is empty.

2 ) If $\varepsilon_{2}=-2 B$ and $\varepsilon_{1}<0, \quad \varepsilon_{1} \leq-\frac{6 B}{W}, \quad$ (respectively, $\varepsilon_{2}=-2 B$ and $\varepsilon_{1}>0, \varepsilon_{1} \geq \frac{6 B}{W}$ ), then the essential spectrum of the operator $\tilde{H}_{3 / 2}^{q}$ is consists of the union of three segments

$$
\begin{aligned}
\sigma_{\text {ess }}\left(\tilde{H}_{3 / 2}^{q}\right)= & {[3 A-18 B, 3 A+18 B] \cup\left[2 A-12 B+z_{1}, 2 A+12 B+z_{1}\right], \text { (respectively, } } \\
& \cup\left[A-6 B+2 z_{1}, A+6 B+2 z_{1}\right] \\
\sigma_{\text {ess }}\left(\tilde{H}_{3 / 2}^{q}\right)= & {[3 A-18 B, 3 A+18 B] \cup\left[2 A-12 B+z_{2}, 2 A+12 B+z_{2}\right], \text { ) and discrete } } \\
& \bigcup\left[A-6 B+2 z_{2}, A+6 B+2 z_{2}\right]
\end{aligned}
$$

spectrum of the operator $\tilde{H}_{3 / 2}^{q}$ is consists of a single eigenvalue, $\sigma_{\text {disc }}\left(\tilde{H}_{3 / 2}^{q}\right)=\left\{3 z_{1}\right\}$, (respectively, $\sigma_{\text {disc }}\left(\tilde{H}_{3 / 2}^{q}\right)=\left\{3 z_{2}\right\}$,) where $z_{1}$ (respectively, $z_{2}$ ) are the eigenvalue of operator $\tilde{H}_{1}$.

If $-\frac{6 B}{W} \leq \varepsilon_{1}<0$ (respectively, $0<\varepsilon_{1} \leq \frac{6 B}{W}$ ), then the essential spectrum of the operator $\tilde{H}_{3 / 2}^{q}$ is consists of a single segment $\sigma_{\text {ess }}\left(\tilde{H}_{3 / 2}^{q}\right)=[3 A-18 B, 3 A+18 B]$, and discrete spectrum of the operator $\tilde{H}_{3 / 2}^{q}$ is empty.

3) If $\varepsilon_{2}=0$ and $\varepsilon_{1}<0, \varepsilon_{1}<-\frac{6 B}{W}$, (respectively, $\varepsilon_{2}=0$ and $\varepsilon_{1}>0, \varepsilon_{1}>\frac{6 B}{W}$ ), then the essential spectrum of the operator $\tilde{H}_{3 / 2}^{q}$ is consists of the union of three segments $\sigma_{\text {ess }}\left(\tilde{H}_{3 / 2}^{q}\right)=[3 A-18 B, 3 A+18 B] \cup[2 A-12 B+z, 2 A+12 B+z]$,

$$
\cup[A-6 B+2 z, A+6 B+2 z]
$$


and discrete spectrum of the operator $\tilde{H}_{3 / 2}^{q}$ is consists of a single point, $\sigma_{\text {disc }}\left(\tilde{H}_{3 / 2}^{q}\right)=\{3 z\}$, where $z$ is the eigenvalue of operator $\tilde{H}_{1}$.

If $\varepsilon_{2}=0$ and $\varepsilon_{1}<0,-\frac{6 B}{W} \leq \varepsilon_{1}<0$, (respectively, $\varepsilon_{2}=0$ and $0<\varepsilon_{1} \leq \frac{6 B}{W}$ ), then the essential spectrum of the operator $\tilde{H}_{3 / 2}^{q}$ is consists of a single segment $\sigma_{\text {ess }}\left(\tilde{H}_{3 / 2}^{q}\right)=[3 A-18 B, 3 A+18 B]$, and discrete spectrum of the operator $\tilde{H}_{3 / 2}^{q}$ is empty set.

4) If $\varepsilon_{1}=0$ and $\varepsilon_{2}>0, E>W$, (respectively, $\varepsilon_{1}=0$ and $\varepsilon_{2}<-2 B, E<W$ ), then the essential spectrum of the operator $\tilde{H}_{3 / 2}^{q}$ is consists of the union of three segments $\sigma_{\text {ess }}\left(\tilde{H}_{3 / 2}^{q}\right)=[3 A-18 B, 3 A+18 B] \cup[2 A-12 B+z, 2 A+12 B+z]$,

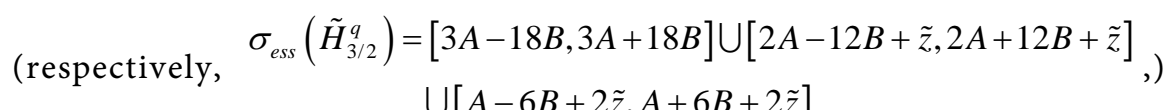
and discrete spectrum of the operator $\tilde{H}_{3 / 2}^{q}$ is consists of a single point, $\sigma_{\text {disc }}\left(\tilde{H}_{3 / 2}^{q}\right)=\{3 z\},\left(\right.$ respectively, $\left.\sigma_{\text {disc }}\left(\tilde{H}_{3 / 2}^{q}\right)=\{3 \tilde{z}\},\right)$ where $z($ respectively, $\tilde{z})$ is the eigenvalue of operator $\tilde{H}_{1}$, and $E=\frac{\left(B+\varepsilon_{2}\right)^{2}}{\varepsilon_{2}^{2}+2 B \varepsilon_{2}}$. If $\varepsilon_{1}=0$ and $\varepsilon_{2}>0, E<W$, (respectively, $\varepsilon_{1}=0$ and $\varepsilon_{2}<-2 B, E>W$ ), then the essential spectrum of the operator $\tilde{H}_{3 / 2}^{q}$ is consists of a single segment $\sigma_{\text {ess }}\left(\tilde{H}_{3 / 2}^{q}\right)=[3 A-18 B, 3 A+18 B]$, and discrete spectrum of the operator $\tilde{H}_{3 / 2}^{q}$ is empty.

5) If $\varepsilon_{1}=\frac{2\left(\varepsilon_{2}^{2}+2 B \varepsilon_{2}\right)}{B}$, (respectively, $\left.\varepsilon_{1}=-\frac{2\left(\varepsilon_{2}^{2}+2 B \varepsilon_{2}\right)}{B}\right)$, then the essential spectrum of the operator $\tilde{H}_{3 / 2}^{q}$ is consists of the union of three segments $\begin{aligned} \sigma_{\text {ess }}\left(\tilde{H}_{3 / 2}^{q}\right)= & {[3 A-18 B, 3 A+18 B] \cup[2 A-12 B+z, 2 A+12 B+z], \quad \text { (respectively, } } \\ & \cup[A-6 B+2 z, A+6 B+2 z] \\ \sigma_{e s s}\left(\tilde{H}_{3 / 2}^{q}\right)= & {[3 A-18 B, 3 A+18 B] \cup[2 A-12 B+\tilde{z}, 2 A+12 B+\tilde{z}], \text {, ) and discrete } } \\ & \bigcup[A-6 B+2 \tilde{z}, A+6 B+2 \tilde{z}]\end{aligned}$ spectrum of the operator $\tilde{H}_{3 / 2}^{q}$ is consists of a single point, $\sigma_{\text {disc }}\left(\tilde{H}_{3 / 2}^{q}\right)=\{3 z\}$, (respectively, $\sigma_{\text {disc }}\left(\tilde{H}_{3 / 2}^{q}\right)=\{3 \tilde{z}\}$,) where $z$ (respectively, $\left.\tilde{z}\right)$ is the eigenvalue of operator $\tilde{H}_{1}$.

6) If $\varepsilon_{2}>0$ and $\varepsilon_{1}>\frac{2\left(\varepsilon_{2}^{2}+2 B \varepsilon_{2}\right)}{B}$ (respectively, $\varepsilon_{2}<-2 B$ and $\left.\varepsilon_{1}>\frac{2\left(\varepsilon_{2}^{2}+2 B \varepsilon_{2}\right)}{B}\right)$, then the essential spectrum of the operator $\tilde{H}_{3 / 2}^{q}$ is consists of the union of three segments

$$
\begin{aligned}
\sigma_{\text {ess }}\left(\tilde{H}_{3 / 2}^{q}\right)= & {[3 A-18 B, 3 A+18 B] \cup\left[2 A-12 B+z_{1}, 2 A+12 B+z_{1}\right], \text { and discrete } } \\
& \cup\left[A-6 B+2 z_{1}, A+6 B+2 z_{1}\right]
\end{aligned}
$$

spectrum of the operator $\tilde{H}_{3 / 2}^{q}$ is consists of a single point, $\sigma_{\text {disc }}\left(\tilde{H}_{3 / 2}^{q}\right)=\left\{3 z_{1}\right\}$, where $z_{1}$ is the eigenvalue of operator $\tilde{H}_{1}$. 
7) If $\varepsilon_{2}>0$ and $\varepsilon_{1}<-\frac{2\left(\varepsilon_{2}^{2}+2 B \varepsilon_{2}\right)}{B}$ (respectively, $\varepsilon_{2}<-2 B$ and $\left.\varepsilon_{1}<-\frac{2\left(\varepsilon_{2}^{2}+2 B \varepsilon_{2}\right)}{B}\right)$, then the essential spectrum of the operator $\tilde{H}_{3 / 2}^{q}$ is consists of the union of three segments

$$
\begin{aligned}
\sigma_{e s s}\left(\tilde{H}_{3 / 2}^{q}\right)= & {[3 A-18 B, 3 A+18 B] \cup\left[2 A-12 B+z_{1}, 2 A+12 B+z_{1}\right], \text { and discrete } } \\
& \cup\left[A-6 B+2 z_{1}, A+6 B+2 z_{1}\right]
\end{aligned}
$$

spectrum of the operator $\tilde{H}_{3 / 2}^{q}$ is consists of a single point, $\sigma_{\text {disc }}\left(\tilde{H}_{3 / 2}^{q}\right)=\left\{3 z_{1}\right\}$, where $z_{1}$ is the eigenvalue of operator $\tilde{H}_{1}$.

8) If $\varepsilon_{2}>0$ and $-\frac{2\left(\varepsilon_{2}^{2}+2 B \varepsilon_{2}\right)}{B}<\varepsilon_{1}<\frac{2\left(\varepsilon_{2}^{2}+2 B \varepsilon_{2}\right)}{B}$ (respectively,

$\varepsilon_{2}<-2 B$ and $\left.-\frac{2\left(\varepsilon_{2}^{2}+2 B \varepsilon_{2}\right)}{B}<\varepsilon_{1}<\frac{2\left(\varepsilon_{2}^{2}+2 B \varepsilon_{2}\right)}{B}\right)$, then the essential spectrum of the operator $\tilde{H}_{3 / 2}^{q}$ is consists of the union of six segments

$\sigma_{\text {ess }}\left(\tilde{H}_{3 / 2}^{q}\right)=[3 A-18 B, 3 A+18 B] \cup\left[2 A-12 B+z_{1}, 2 A+12 B+z_{1}\right]$

$\cup\left[2 A-12 B+z_{2}, 2 A+12 B+z_{2}\right] \cup\left[A-6 B+2 z_{1}, A+6 B+2 z_{1}\right] \quad$ and discrete

$\cup\left[A-6 B+z_{1}+z_{2}, A+6 B+z_{1}+z_{2}\right] \cup\left[A-6 B+2 z_{2}, A+6 B+2 z_{2}\right]$

spectrum of the operator $\tilde{H}_{3 / 2}^{q}$ is consists of a four points,

$\sigma_{\text {disc }}\left(\tilde{H}_{3 / 2}^{q}\right)=\left\{3 z_{1}, 2 z_{1}+z_{2}, z_{1}+2 z_{2}, 3 z_{2}\right\}$, where $z_{1}$, and $z_{2}$, are the eigenvalues of operator $\tilde{H}_{1}$.

9) If $-2 B<\varepsilon_{2}<0$, then the essential spectrum of the operator $\tilde{H}_{3 / 2}^{q}$ is consists of a single segment $\sigma_{\text {ess }}\left(\tilde{H}_{3 / 2}^{q}\right)=[3 A-18 B, 3 A+18 B]$, and discrete spectrum of the operator $\tilde{H}_{3 / 2}^{q}$ is empty set.

Proof. The proof of Theorems 9 is similar to the proof of Theorems 8 .

\section{Conclusions}

In this paper, we investigated the structure of essential spectra and discrete spectrum of the three-electron systems in the impurity Hubbard model in the quartet state of the system. In the investigation of the spectra of three-electron systems in the impurity Hubbard model, we will reduce to a study the spectrum of a simpler operator. The paper consists of five sections. In the first section called Introduction, we give a summary historical description of the problem, and also describe the Hamiltonian of the considering systems. In the second section called Quartet state, we give the basic function of the system in the quartet state, also describe the action of the three-electron quartet state operator in the coordinate representation in the invariant subspace. This operator is the bounded self-adjoint operator. Using the Fourier transform, we find the action of the three-electron quartet state operator in a quasimomentum representation. This operator is a bounded self-adjoint operator. In the third section of the paper, we describe the spectrum of the energy operator of a one-electron system in the impurity Hubbard model in a $v$-dimensional integer-valued lattice $Z^{v}$. The 
Hamiltonian of one-electron systems in the impurity Hubbard model also has the form (1), but now the operator acts in the space one-electron states $\mathscr{H}_{1}$. We denote by $H_{1}$ the restriction of $H$ to the space $\mathscr{H}_{1}$. We find the action of the operator $H_{1}$ in the coordinate and quasimomentum representations. The operator $H_{1}$ and $\tilde{H}_{1}$ are bounded self-adjoint operators. The operator $\tilde{H}_{1}$ has a continuous spectrum and a finite number of eigenvalues with finite multiplicities. The finding of the eigenvalues of the operator $\tilde{H}_{1}$ is reduced to finding zeros of the function $\Delta_{v}(z)$.

We showed that the operator $\tilde{H}_{1}$, in addition to the continuous spectrum, has or one eigenvalue below the continuous spectrum, or one eigenvalue above the continuous spectrum, or two eigenvalues, of which one is below and the other is above than the continuous spectrum, or there are no eigenvalues.

Using tensor products of Hilbert spaces and tensor products of operators in Hilbert spaces, we can verify that the three-electron quartet state operator $\tilde{H}_{3 / 2}^{q}$ can be represented in the form

$$
\tilde{H}_{3 / 2}^{q}=\tilde{H}_{1} \otimes I \otimes I+I \otimes \tilde{H}_{1} \otimes I+I \otimes I \otimes \tilde{H}_{1},
$$

where $I$ is the unit operator in the space $\mathscr{H}_{1}$.

Now, using the obtained results on the spectrum of the operator $\tilde{H}_{1}$ and this representation, we can describe the structure of the essential spectrum and the discrete spectrum of the three-electron quartet state operator $\tilde{H}_{3 / 2}^{q}$.

We prove that the essential spectrum of the three-electron quartet state operator $\tilde{H}_{3 / 2}^{q}$ is consists or of a single segment, or of the union of three segments, or of the union of six segments, and the discrete spectrum of the three-electron quartet state operator $\tilde{H}_{3 / 2}^{q}$ consists of an empty set, or of a single eigenvalue, or of four eigenvalues.

\section{Conflicts of Interest}

The author declares no conflicts of interest regarding the publication of this paper.

\section{References}

[1] Hubbard, J. (1963) Electron Correlations in Narrow Energy Band. Proceedings of the Royal Society A, 276, 238-257. https://doi.org/10.1098/rspa.1963.0204

[2] Gutzwiller, M.C. (1963) Effect of Correlation on the Ferromagnetism of Transition Metals. Physical Review Letters, 10,159-162. https://doi.org/10.1103/PhysRevLett.10.159

[3] Kanamori, J. (1963) Electron Correlation and Ferromagnetism of Transition Metals. Progress of Theoretical Physics, 30, 275-289. https://doi.org/10.1143/PTP.30.275

[4] Anderson, P.W. (1961) Localized Magnetic States in Metals. Physical Review Journals Archive, 124, 41-53. https://doi.org/10.1103/PhysRev.124.41

[5] Shubin, S.P. and Wonsowsky, S.V. (1934) On the Electron Theory of Metals. Proceedings of the Royal Society A, 145, 159-180.

https://doi.org/10.1098/rspa.1934.0089

[6] Karpenko, B.V., Dyakin, V.V. and Budrina, G.L. (1986) Two Electrons in the Hub- 
bard Model. Physics of Metals and Metallography, 61, 702-706.

[7] Mattis, D. (1986) The Few-Body Problems on a Lattice. Reviews of Modern Physics, 58, 361-379. https://doi.org/10.1103/RevModPhys.58.361

[8] Lieb, E. (1989) Two Theorems on the Hubbard Model. Physical Review Letters, 62, 1201-1204. https://doi.org/10.1103/PhysRevLett.62.1201

[9] Tsvelick A. M. and Wiegman P. B. (1983) Exact Results in the Theory of Magnetic Alloys. Advances in Physics, 32, 453-713.

https://doi.org/10.1080/00018738300101581

[10] Izyumov, Y.A. and Skryabin, Y.N. (1987) Statistical Mechanics of Magnetically Ordered Systems. Nauka, Moscow. (In Russian)

[11] Tashpulatov, S.M. (2014) Spectral Properties of Three-Electron Systems in the Hubbard Model. Theoretical and Mathematical Physics, 79, 712-728. https://doi.org/10.1007/s11232-014-0173-y

[12] Tashpulatov, S.M. (2016) Spectra of the Energy Operator of Four-Electron Systems in the Triplet State in the Hubbard Model. Journal of Physics: Conference Series, 697, Article No. 012025. https://doi.org/10.1088/1742-6596/697/1/012025

[13] Tashpulatov, S.M. (2017) The Structure of Essential Spectra and Discrete Spectrum of Four-Electron Systems in the Hubbard Model in a Singlet State. Lobachevskii Journal of Mathematics, 38, 530-541. https://doi.org/10.1134/S1995080217030246

[14] Reed, M. and Simon, B. (1978) Methods of Modern Mathetical Physics. Vol. 1, Functional Analysis. Academic Press, New York.

[15] Valkov, V.V., Ovchinnikov, S.G. and Petrakovskii, O.P. (1986) The Excitation Spectra of Two-Magnon Systems in Easy-Axis Quasidimensional Ferromagnets. Sov. Phys. Solid State, 30, 3044-3047.

[16] Reed, M. and Simon, B. (1982) Methods of Modern Mathetical Physics, Vol. 4, Operator Analysis. Academic Press, New York.

[17] Ichinose, T. (1978) Spectral Properties of Tensor Products of Linear Operators. I. Transactions of the American Mathematical Society, 235, 75-113. https://doi.org/10.1090/S0002-9947-1978-0472915-2

[18] Ichinose, T. (1978) Spectral Properties of Tensor Products of Linear Operators. II: The Approximate Point Spectrum and Kato Essential Spectrum. Transactions of the American Mathematical Society, 237, 223-254. https://doi.org/10.2307/1997620

[19] Ichinose, T. (1982) On the Spectral Properties of Tensor Products of Linear Operators in Banach Spaces. Banach Center Publications, Vol. 8, PWN-Polish Scientific Publishers, Warsaw, 295-300. https://doi.org/10.4064/-8-1-295-300 